\title{
Long noncoding RNA LINC00518 induces radioresistance by regulating glycolysis through an miR-33a-3p/HIF-1a negative feedback loop in melanoma
}

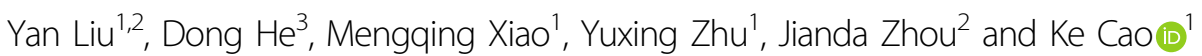

\begin{abstract}
The long noncoding RNA, LINCO0518, is highly expressed in various types of cancers and is involved in cancer progression. Although LINC00518 promotes the metastasis of cutaneous malignant melanoma (CMM), the mechanism underlaying its effects on CMM radiosensitivity remains unclear. In this study, LINC00518 expression was significantly upregulated in CMM samples, and LINC00518 levels were associated with poor prognosis of patients with CMM. Knockdown of LINC00518 in CMM cells significantly inhibited cell invasion, migration, proliferation, and clonogenicity. LINC00518-mediated invasion, migration, proliferation, and clonogenicity were negatively regulated by the microRNA, miR-33a-3p, in vitro, which increased sensitivity to radiotherapy via inhibition of the hypoxia-inducible factor $1 a$ (HIF-1a)/lactate dehydrogenase A glycolysis axis. Additionally, HIF-1a recognized the miR-33a-3p promoter region and recruited histone deacetylase 2, which decreased the expression of miR-33a-3p and formed an LINC00518/miR-33a-3p/ HIF-1a negative feedback loop. Furthermore, signaling with initially activated glycolysis and radioresistance in CMM cells was impaired by Santacruzamate A, a histone deacetylase inhibitor, and 2-deoxy-D-glucose, a glycolytic inhibitor. Lastly, knockdown of LINC00518 expression sensitized CMM cancer cells to radiotherapy in an in vivo subcutaneously implanted tumor model. In conclusion, LINC00518 was confirmed to be an oncogene in CMM, which induces radioresistance by regulating glycolysis through an miR-33a-3p/HIF-1a negative feedback loop. Our study, may provide a potential strategy to improve the treatment outcome of radiotherapy in CMM.
\end{abstract}

\section{Introduction}

Cutaneous malignant melanoma (CMM), a common skin malignancy with rapid progression and poor prognosis, is responsible for $\sim 75 \%$ of all skin tumor mortalities $^{1,2}$. Despite advances in treatment strategies, CMM recurs in $\sim 75 \%$ of patients, 1 year following treatment; the 3-year overall survival (OS) rate of terminal CMM patients is no more than $30 \%^{3-5}$. Radiotherapy treatment

\footnotetext{
Correspondence: Ke Cao (csucaoke@163.com)

'Department of Oncology, Third Xiangya Hospital, Central South University,

Changsha, 410013 Hunan, PR China

${ }^{2}$ Department of Plastic Surgery, Third Xiangya Hospital, Central South

University, Changsha, 410013, PR China

Full list of author information is available at the end of the article

Edited by G. Calin
}

is available for certain primary CMMs, and adjuvant radiotherapy is necessary for infiltrating neutrophilic melanoma, metastatic encephaloma, and bone metas$\operatorname{tases}^{6,7}$. Palliative radiotherapy significantly mitigates symptoms such as bone pain and central nervous system dysfunction induced by CMM metastases ${ }^{8,9}$. However, radioresistance limits the clinical application of radiotherapy, which attributes to poor prognosis. Thus, overcoming radioresistance of CMM could improve therapeutic outcomes of patients with CMM.

Hypoxia, a common phenomenon in solid tumors, is a prognostic indicator for radiotherapy outcomes ${ }^{10,11}$. Hypoxia plays a significant role in radioresistance due reduced cell viability and fixation of DNA damage ${ }^{12,13}$.

\section{(c) The Author(s) 2021}

(c) Open Access This article is licensed under a Creative Commons Attribution 4.0 International License, which permits use, sharing, adaptation, distribution and reproduction cc) in any medium or format, as long as you give appropriate credit to the original author(s) and the source, provide a link to the Creative Commons license, and indicate if changes were made. The images or other third party material in this article are included in the article's Creative Commons license, unless indicated otherwise in a credit line to the material. If material is not included in the article's Creative Commons license and your intended use is not permitted by statutory regulation or exceeds the permitted use, you will need to obtain permission directly from the copyright holder. To view a copy of this license, visit http://creativecommons.org/licenses/by/4.0/. 
In a hypoxic microenvironment, cancer cells obtain energy primarily by inhibiting aerobic respiration and promoting glycolysis and tricarboxylic acid (TCA) cycle ${ }^{14}$. Several glycolysis- and metabolism-related proteins participate in the molecular mechanisms underlaying radioresistance. Hypoxia-inducible factor $1 \alpha$ (HIF- $1 \alpha)$ aggravates the transformation of normal melanocytes into melanoma ${ }^{15,16}$. Lactate dehydrogenase isoform A (LDHA), a key HIF-1 $\alpha$ target, catalyzes the reduction of pyruvate to lactate and sustains cell survival under hypoxic conditions by compensating for the reduction in oxidative mitochondrial functions ${ }^{17}$. The HIF- $1 \alpha /$ LDHA pathway is involved in tumor-protective responses against radiotherapy ${ }^{18}$.

Targeting tumor glucose metabolism and HIF- $1 \alpha$ could alter tumor microenvironment, leading to metabolic alterations and sensitization of multiple solid cancers to radiotherap ${ }^{19}$. Additionally, previous studies have found that the levels of certain glycolysis-related proteins are closely associated with radiotherapy resistance; for instance, higher expression of glucose transporter 1 in breast and liver cancers indicates poor prognosis of patients treated with radiotherapy $y^{20,21}$. Hexokinase 2 (HK2) is a critical rate-limiting enzyme in the glycolytic pathway. Multiple regression analysis models showed that HK2 was an independent negative prognostic factor for cervical squamous carcinoma ${ }^{22}$.

Long noncoding RNAs (lncRNAs), an important class of noncoding RNAs of $>200$ bases with limited protein coding capability, are closely correlated with the occurrence and development of human diseases ${ }^{23,24}$. LncRNAs participate in a series of modifications of the glycolysis pathway in cancer cells: LncRNA IDH1-AS1 links the functions of MYC proto-oncogene, BHLH transcription factor (c-MYC), and HIF- $1 \alpha$ via isocitrate dehydrogenase $(\mathrm{NADP}(+))$ 1, cytosolic (IDH1), to the regulation of mitochondrial respiration and glycolysis in cervical carcinoma cells. Restoring IDH1-AS1 expression might provide a potential metabolic approach to treat cervical carcinoma ${ }^{25}$. LncRNA-p21 is a hypoxiaresponsive lncRNA that induces HIF- $1 \alpha$ accumulation by integrating HIF- $1 \alpha$ and Von Hippel-Lindau tumor suppressor (VHL), thus interrupting the VHL-HIF-1 $\alpha$ interaction, which promotes glycolysis under hypoxic conditions in colorectal cancer cells ${ }^{26}$. Studies have shown that InRNAs, such as LncRNA-TUG1 and $L n c R N A-M I F$, participate in the glycolytic pathway and regulate glycol-metabolism via competing endogenous RNA (ceRNA) networks ${ }^{27-30}$.

To date, no studies have demonstrated the role of lncTNAs in regulating CMM radiosensitivity. In the present study, we analyzed two CMM data sets, GSE46517 $7^{31}$ and GSE4587 ${ }^{32}$ published in the Gene Expression Omnibus (GEO) database, to investigate dysregulated lncRNAs in CMM. Long intergenic noncoding RNA 518 (LINC00518) was significantly upregulated in CMM tissues compared with that in normal tissues, which promotes the metastasis of malignant melanoma ${ }^{33}$. The Cancer Genome Atlas (TCGA) data set showed that high expression of LINC00518 indicated a worse relapse-free survival (RFS) and OS of patients with CMM. Furthermore, we verified that miR-33a-3p could form a negative feedback loop with HIF- $1 \alpha$ and increase radiosensitivity of CMM by modulating the glycolytic pathway. The results of the present study provide a new perspective on the role of lncRNAs in radiation sensitivity of CMM and may help to identify new biomarkers or targeted therapies for CMM.

\section{Results}

LINC00518 is overexpressed in CMM and indicates poor prognosis in patients with CMM

To identify novel lncRNAs in CMM, we have downloaded two cohorts of gene expression data sets for CMM from the GEO database (GSE46517 and GSE4587). The SAM software was used to analyze differences in lncRNA expression in biopsy samples of CMM and normal skin tissues. We identified 12 lncRNAs that expressions were increased in CMM compared with normal skin tissues (Fig. 1A). Among the differentially expressed lncRNAs, LINC00518 was overexpressed in the CMM samples from both data sets (Fig. 1B). Based on data from the TCGA database, we found that the expression level of LINC00518 was higher in CMM than in non-CMM samples (Fig. 1C). Patients with CMM who showed high expression of LINC00518 had a significantly shorter OS $(P=0.0009$, Fig. $1 C)$ and RFS $(P=0.038$, Fig. $1 C)$. This result shows that the expression of LINC00518 was increased in patients with CMM, and this high expression is associated with poor survival of these patients.

\section{LINC00518 knockdown suppressed cell proliferation, colony formation, migration and invasion, and induced apoptosis}

LINC00518 was upregulated in CMM tissues compared with normal tissues in GSE46517 and GSE4587 data sets (Fig. 2A). Subsequently, LINC00518 expression was confirmed in 12 paired CMM tissues and their corresponding normal skin tissues using quantitative real-time reverse transcription PCR (qRT-PCR) (Fig. 2B). To investigate the function of LINC00518 in CMM, we analyzed LINC00518 expression in several CMM cell lines (human melanocytes (HM), WM35, WM451, and A375). LINC00518 expression was higher in metastatic melanoma cell lines (WM451 and A375 cells), and lower in normal melanocytes cell lines (HM) and primary melanoma cell lines (WM35 cells) (Fig. 2C), these melanoma cell lines have shown different metastatic potential ${ }^{34,35}$. Therefore, we 
A

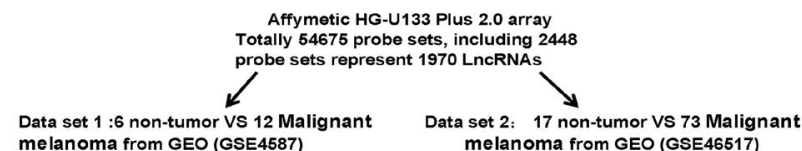

melanoma from GEO (GSE4587) melanoma from GEO (GSE46517)

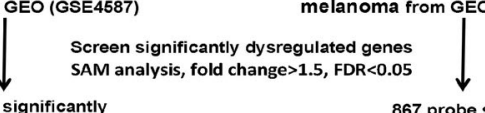

2408 probe sets significantly $\quad 12$ probe sets represent $\quad 867$ probe sets significantly

differentiate expression 12 probe sets represent differentiate expression

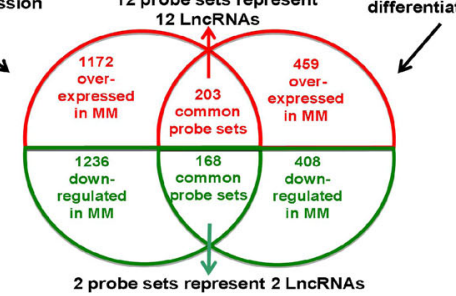

GSE4587

GSE46517

B

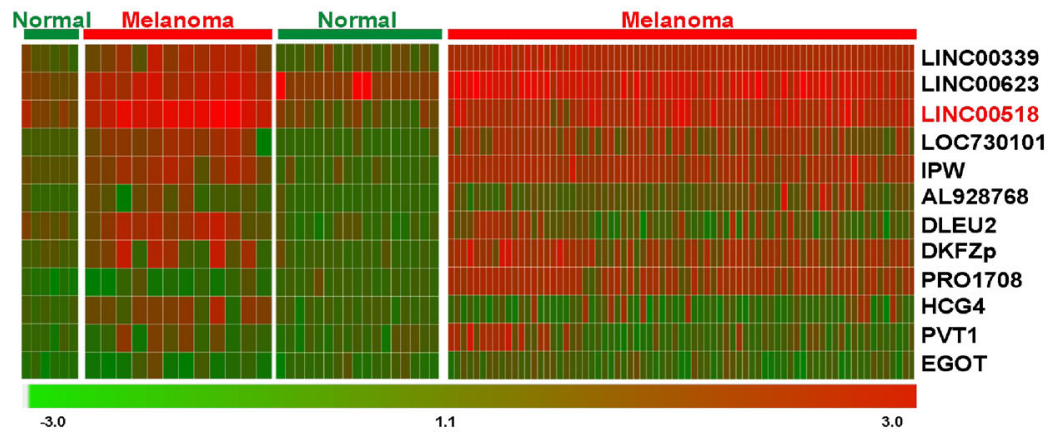

C
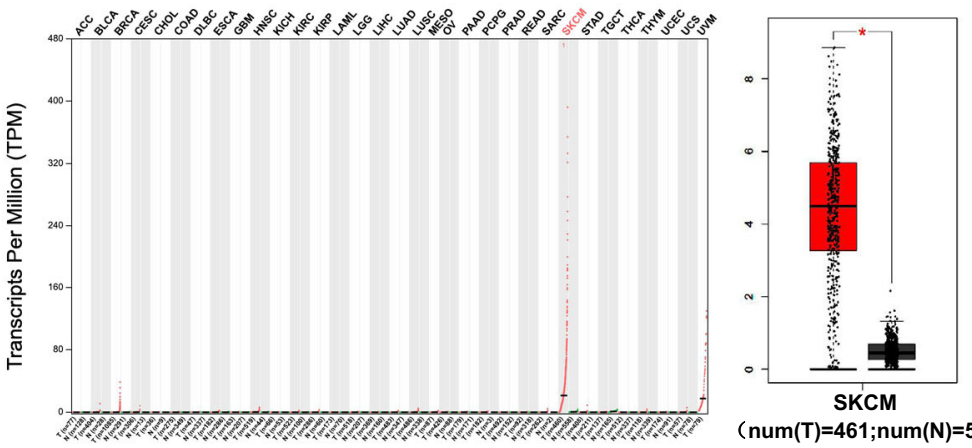

( $\operatorname{num}(T)=461 ; \operatorname{num}(N)=558)$

D
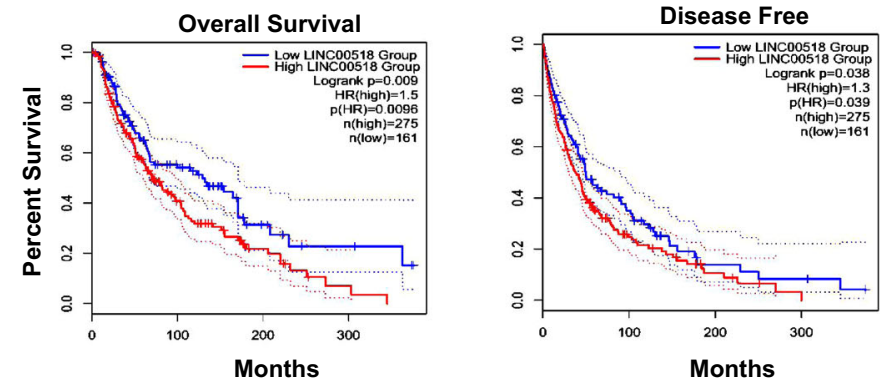

Fig. 1 LINC00518 is overexpressed in CMM and indicates poor prognosis in patients with CMM. A Schematic overview of the workflow used to identify and validate dysregulated IncRNAs in two CMM microarray data cohorts. B Heatmap of 12 upregulated probe sets representing 12 IncRNAs mined from the GSE4587 and GSE46517 data sets. C TCGA data showing that the Transcripts Per Million of LINC00518 in CMM was highest in all human cancers and that it is upregulated in CMM compared with normal tissues. D Kaplan-Meier curves of the OS and RFS of 436 patients with CMM with high or low LINC00518 expression. P value was computed by the log-rank test. SKCM stands for skin cutaneous melanoma. 


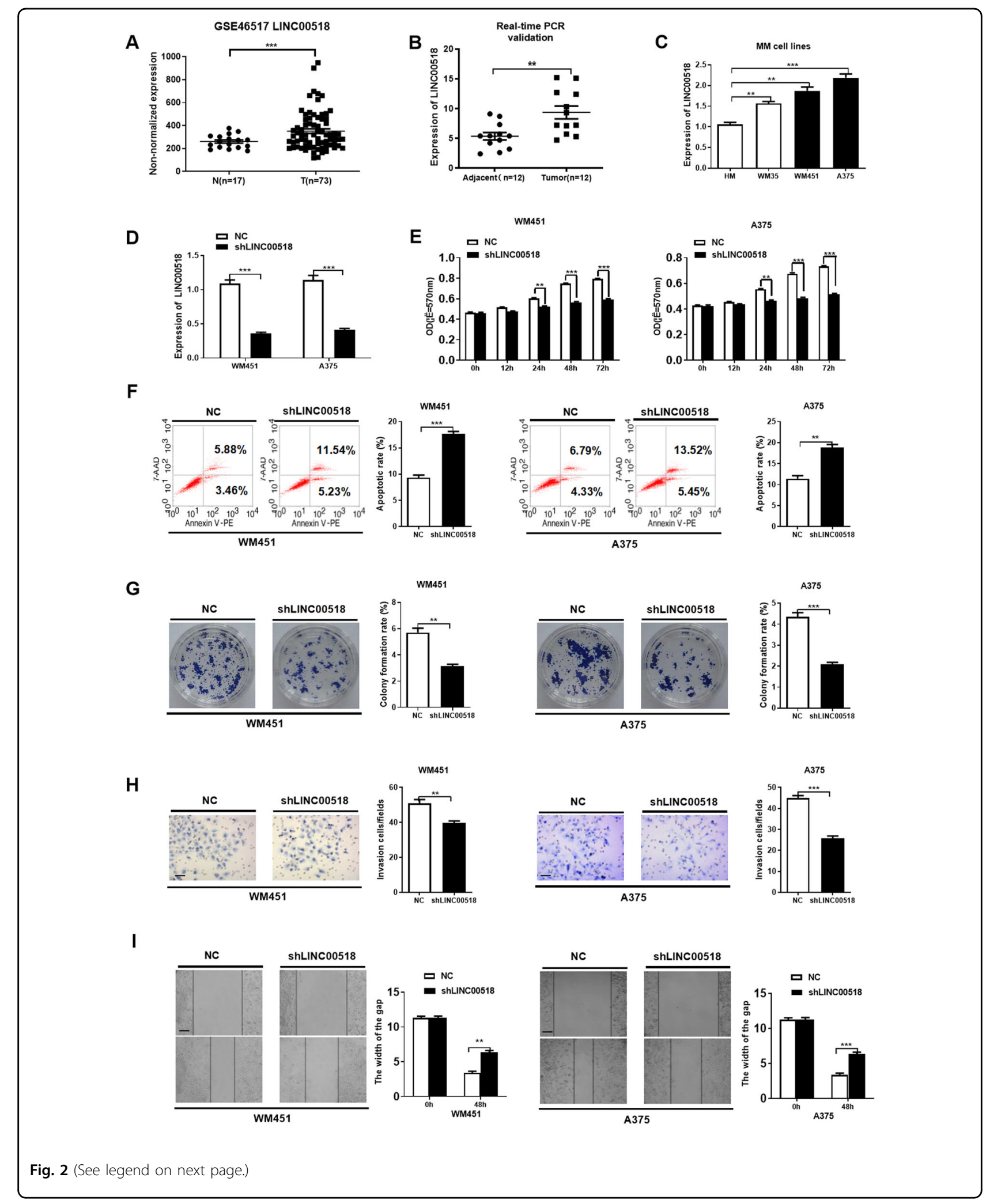

knocked down LINC00518 expression using a LINC00518-targeting small hairpin RNA (shRNA) in metastatic melanoma cell lines, WM451 and A375
(Fig. 2D). Next, we conducted phenotypic and functional analyses of the effects of LINC00518 knockdown in WM451 and A375 cell lines after establishing shRNA 
(see figure on previous page)

Fig. 2 LINC00518 knockdown suppressed cell proliferation, colony formation, migration and invasion, and reduced viability. A The gene chip data related to LINC00518 were upregulated in T group (CMM tissues, $n=73$ ) compared with N group in GSE46517 data set (normal tissues, $n=$ 17). B qPCR assay showing that LINC00518 was overexpressed in 12 paired CMM tissues and their corresponding adjacent noncancerous skin tissues obtained from tissue biopsy samples in our study. C qPCR assay showing that LINC00518 expression was higher in WM451 and A375 cells, which have high invasive and metastatic tendency; however, it was lower in HM (normal melanocyte cell lines) and WM35 (low invasive and metastatic tendency) cells. The expression data in HM were considered as a control. D qPCR assay demonstrating shLINC00518 knockdown of LINC00518 in WM451 and A375 cell lines. E MTT assay indicating that LINC00518 knockdown in WM451/A375 cells decreased viability significantly compared with that in control cells. F Flow cytometry examination showing that LINC00518 knockdown could increase apoptosis of CMM cells. G Colony formation assay indicating that knockdown of LINC00518 could suppress cell proliferation in CMM cells. $\mathbf{H}$ Transwell assay showing the effect on CMM cell migration following LINC00518 knockdown. I Wound-healing assay showing that LINC00518 knockdown in WM451/A375 cells can significantly inhibit cell migration compared with the control. The histogram data for each group are an average of three independent replicates; bars indicate $S D ;{ }^{*} P<0.05$, ${ }^{* *} P<0.01$, ***P $<<0.001$.

efficacy. 3-(4,5-dimethylthiazol-2-yl)-2,5-diphenyltetrazolium bromide (MTT) assay showed that the proliferation of LINC00518-silenced cells was significantly decreased compared to that of control CMM cells (Fig. 2E). Flow cytometry demonstrated that silencing of LINC00518 expression in WM451 and A375 cells increased apoptosis (Fig. 2F), while colony formation assays indicated that the cell survival capability of LINC00518-silenced cells significantly decreased (Fig. 2G). The effect of LINC00518 on the migration and invasion of CMM cells was measured using wound-healing and Transwell Matrigel invasion assays, respectively. LINC00518 shRNA-transfected CMM cells displayed lower migratory and invasive capabilities than the control cells (Fig. 2H, I). Our results suggest that LINC00518 could promote metastasis in malignant melanoma.

\section{LINC00518 directly targets miR-33a-3p and promotes HIF- 1a expression}

LINC00518 influences several human tumors through the ceRNA mechanism ${ }^{36,37}$. To explore the mechanisms of LINC00518 in CMM tumorigenesis, we initially conducted KEGG pathway analysis using TCGA database. The results showed that LINC00518 may play a role in glycolysis and TCA cycle (Fig. 3A). Subsequently, miRNA target prediction databases RegRNA (http://regrna2.mbc. nctu.edu.tw/) and TargetScan (www.targetscan.org) were used to predict miRNAs, which LINC00518 could sponge, and the target genes, which miRNAs could regulate (Fig. 3B). In the network, miR-33a-3p expression was most significantly increased by shLINC00518 among all miRNAs that LINCO0518 could sponge (Fig. 3C), and it may target HIF- $1 \alpha$. HIF- $1 \alpha$ is a known oncogenic gene that accelerate glycolysis in cancer cells and is involved in the occurrence and development of $\mathrm{CMM}^{16,38}$. To validate the results of ceRNA analysis, the expression of miR$33 a-3 p$ and HIF- $1 \alpha$ was measured in CMM cells line. MiR-33a-3p was decreased in WM451 and A375 cells, which have a high invasive and metastatic tendency, while HIF- $1 \alpha$ was increased in melanoma WM451 and A375 cells (Fig. 3D, E). Next, presumptive binding sites for miR33a-3p were identified in the LINCO0518 sequence and in
HIF-1 $\alpha$ 3UTR using RegRNA 2.0 website (Fig. 3F). Expression of miR-33a-3p in CMM biopsy tissues compared with that in normal tissues was assessed, and an inverse correlation between miR-33a-3p and LINC00518 expression was observed (Fig. 3G). Results of RNA pulldown demonstrated that the bio-miR-33a-3p group exhibited a larger amount of LINC00518 than the biomiR-NC and bio-miR-33a-3p-MUT (mutant) groups (Fig. 3H). We used dual-luciferase experiments to determine whether LINC00518 could directly target miR-33a$3 p$. These assays demonstrated that miR-33a-3p mimics significantly inhibited the relative dual-luciferase activity of the LINC00518-WT (wild type) group compared with that of control group, while they did not affect the reporter activity of LINC00518-MUT group (Fig. 3I). These results indicated that LINC00518 could directly target miR-33a-3p and reduce its expression.

In addition, correlation analysis showed a negative correlation between miR-33a-3p and HIF- $1 \alpha$ expressions in CMM biopsy tissues (Fig. 3J). The significant inhibition of the relative dual-luciferase activity of the HIF- $1 \alpha-$ WT group by miR-33a-3p mimics and shLINC00518 indicated that miR-33a-3p could regulate HIF- $1 \alpha$ expression (Fig. 3K). qRT-PCR indicated that HIF- $1 \alpha$ expression was decreased by transfected with shLINC00518 and miR33a-3p mimic in WM451 and A375, and the effect of shLINC00518 can be reversed by miR-33a-3p inhibitor. To explore the effect of LINC00518 knockdown and miR$33 a-3 p$ on glycolysis, we examined changes in the abundance of HIF- $1 \alpha$ and LDHA proteins in CMM cell line. Western blotting analysis indicated that HIF- $1 \alpha$ and LDHA protein levels were decreased by transfected with shLINC00518 and miR-33a-3p mimic (Fig. 3L, M). These results indicated that LINC00518 increased the expression of HIF-1 $\alpha$ in CMM by sponging miR-33a-3p.

\section{HIF-1a negatively regulates miR-33a-3p expression in CMM cells}

HIF- $1 \alpha$ can bind to a promoter to regulate gene expression levels. JASPAR server predicted it presumptive binding sites at positions $632-639 \mathrm{bp}$ in a sequence upstream of the 
A
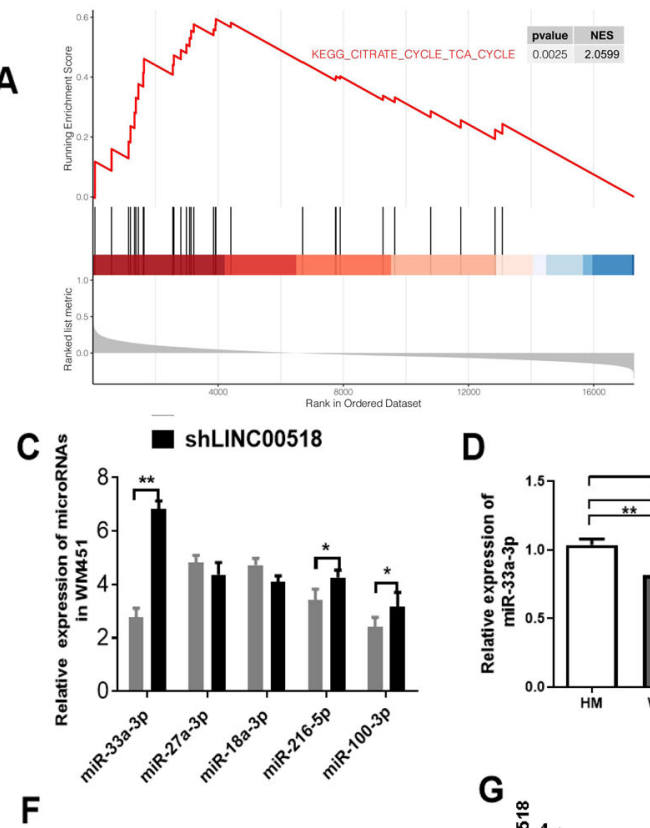

LINC00518 5-AAAAAGTTTTAGTCACTGTGATTCCATTT-3 miR-33a-3p 3-CACTACGTIIIII HIF-1 $\alpha$ 5-ATGGAAGGTATTGCACTGCACA-3

D

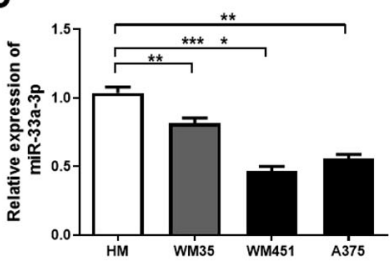

G

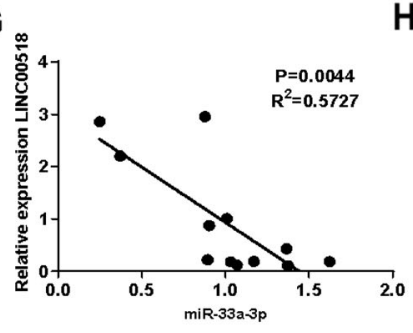

I

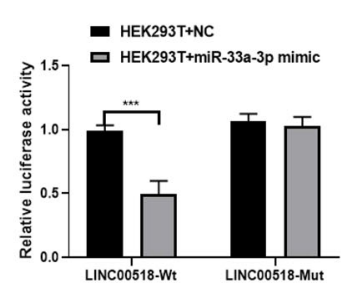

B

E
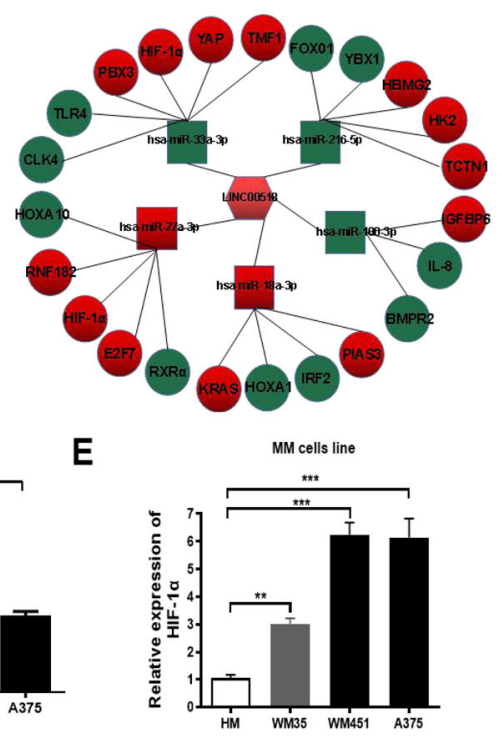

H

- WM451+Bio-miR-NC WM451+Bio-miR-33a-3p A375+Bio-miR-NC

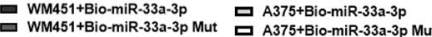
WM451+Bio-miR-33a-3p Mut 口 A375+Bio-miR-33a-3p Mu

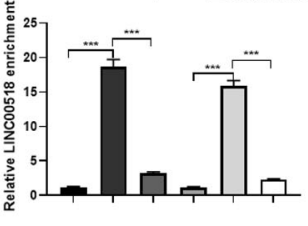

- HEK293T+NC

- HEK293T+miR-33a-3p mimic

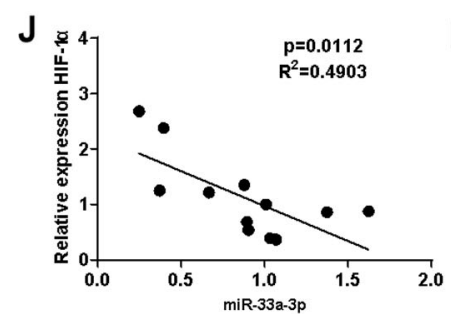

K

ㅁ HEK293T+shLINC00518

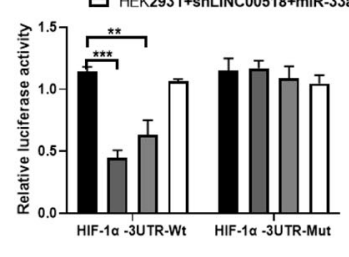

L

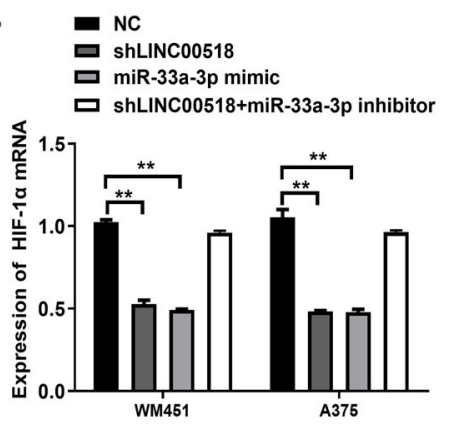

M

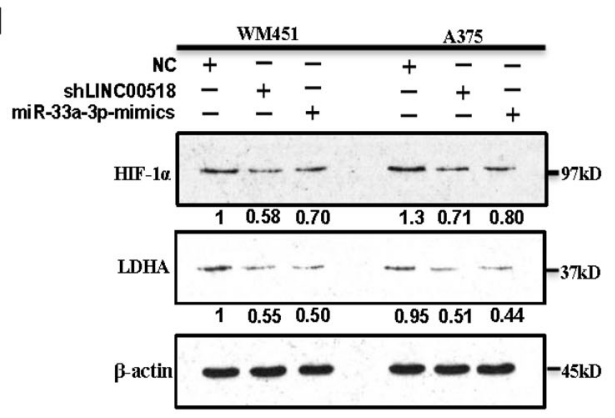

Fig. 3 (See legend on next page.)

origin of miR-33a, with a score of 7.1 out of 10 (Fig. 4A), therefore, we speculated that HIF- $1 \alpha$ could bind to the miR-33a-3p promoter and regulate its expression levels.
PCR assays demonstrated that miR-33a-3p expression was elevated after LINC00518 and HIF-1 $\alpha$ knockdown in WM451 and A375 cell lines. We observed that enhanced 
(see figure on previous page)

Fig. 3 LINC00518 directly targets miR-33a-3p and promotes HIF-1a expression. A Kegg pathway analysis showed that LINC00518 may play a major role in glycolysis and TCA cycle. B Cytoscape was used to visualize LINC00518-miRNA-target gene interactions. The LINC00518/miRNAs interaction was predicted using RegRNA 2.0 and miRbase, the target genes of the miRNAs were predicted by combing GEO and TCGA databases with miRNA target prediction tools (microRNA.org and TargetScan). Red color demonstrates high expression level in CMM, and blue color demonstrates low expression level. C The expression levels of miR-33a-3p, miR-27a-3p, miR-18a-3p, miR216-5p, and miR-100-3p in melanoma cells following transfection with LINC00518 shRNA or NC. D The miR-33a-3p expression profile in human melanoma cell lines (WM35, WM451, A375) and human epidermal melanocytes (HM). E The HIF-1a expression profile in human melanoma cell lines (WM35, WM451, A375) and human epidermal melanocytes (HM). F Presumptive binding sites for miR-33a-3p were identified in the LINC00518 sequence and in the HIF-1a $3^{\prime}$ UTR by using sequence comparison and RegRNA data. G Negative correlation between LINC00518 and miR-33a-3p expressions in 12 cases of CMM tissues using Pearson correlation analysis. H RNA pull-down depicting that the bio-miR-33a-3p group was enriched for larger amounts of LINC00518 than the biomiR-NC and bio-miR-33a-3p-MUT groups, thereby indicating that LINC00518 can target miR-33a-3p directly. I A dual-luciferase assay showing that miR-33a-3p mimics directly and significantly inhibited the relative dual-luciferase activity of the LINC00518-WT group compared with that of control group and LINC00518 targeted miR-33a-3p. Luciferase activity was detected $48 \mathrm{~h}$ after transfection. J qPCR assay showing a negative correlation between miR-33a-3p and HIF-1a expressions in 12 cases of CMM tissues. $\mathbf{K}$ Dual-luciferase assay of cells transfected with HIF-1a-3'UTR-WT or HIF-1a3'UTR-MUT reporter together with miR-33a-3p mimic, LINC00518 shRNA, or LINC00518 shRNA plus miR-33a-3p inhibitor. L The changes of HIF-1a mRNA expression in WM451 and A375 transfected with miR-33a-3p mimic, LINC00518 shRNA, or LINC00518 shRNA plus miR-33a-3p inhibitor. M Western blotting analysis showing that HIF-1a and LDHA protein levels were regulated by LINC00518 and miR-33a-3p. The histogram data for each group are an average of three independent replicates; bars indicate $\mathrm{SD}$; ${ }^{*} P<0.05,{ }^{* *} P<0.01$, ${ }^{* *} P<0.001$.

HIF-1 $\alpha$ expression in WM451 and A375 cells could rescue the effect on miR-33a-3p expression by LINC00518 knockdown (Fig. 4C, D). Furthermore, LINC00518 expression was increased when HIF-1 $\alpha$ expression was enhanced in LINC00518 knockdown CMM cells (Fig. 4E). Results indicate that HIF- $1 \alpha$ could negatively regulate miR-33a-3p expression. Dual-luciferase assay also showed that HIF-1 $\alpha$ could bind to the promoter region of miR-33a-3p. Chromatin immunoprecipitation (ChIP) PCR was conducted to verify the results, where HIF- $1 \alpha$ was overexpressed or inhibited in WM451 cells and anti-HIF-1 $\alpha$ antibody was used to precipitate complexes prior to DNA extraction. Primers were designed for the miR-33a-3p promoter regions, and PCR was conducted to detect miR33a-3p expression levels. Overexpression of HIF- $1 \alpha$ in WM451 cells had significantly deceased miR-33a-3p enrichment, whereas HIF-1 $\alpha$-silenced WM451 cells had significantly decreased miR-33a-3p enrichment. These results confirmed that HIF- $1 \alpha$ could bind to the miR-33a3p promoter but inhibited its expression.

Histone deacetylases (HDACs) are important components of protein complexes that affect the dynamics of chromatin folding during gene transcription. The combination of HDACs with transcription factors that bind to a promoter could inhibit gene expression by accelerating histone deacetylation ${ }^{39}$. The PCR results indicated that the silencing of histone deacetylase 1 (HDAC1) and histone deacetylase 2 (HDAC2) significantly increased miR33a-3p expression. A co-immunoprecipitation assay showed that the HIF-1 $\alpha-$ HDAC immunoprecipitated was decreased following HIF-1 $\alpha$ knockdown and increased following HIF-1 $\alpha$ overexpression, indicated that HDAC1 and 2 can bind to HIF-1 $\alpha$. Moreover, ChIP PCR was conducted to verify that Santacruzamate A, an inhibitor of HDACs, could recover the relative expression levels of miR-33a-3p in WM451 and A375 cells (Fig. 4G).
Western blotting analysis indicated that LINC00518 knockdown decreased LDHA protein levels, while overexpression of HIF- $1 \alpha$ in LINC00518 knockdown cells enhanced HIF- $1 \alpha$ and LDHA expression, suggesting that a LINC00518-HIF- $1 \alpha$ axis exists in CMM cells (Fig. 4J). These results indicated that HIF- $1 \alpha$ could regulate miR33a-3p expression through a negative feedback mechanism. It is possible that HIF- $1 \alpha$ could bind to the miR-33a promoter and repress the transcription of miR-33a by accelerating histone deacetylation of miR-33a.

\section{Overexpression of HIF-1a reversed LINC00518 knockdown- induced suppressing of proliferation, migration, invasion, and colony formation}

LINC00518 regulates proliferation, apoptosis, colony formation, migration, and invasion in CMM cells, and directly inhibits the expression of miR-33a-3p and then increase of HIF- $1 \alpha$ expression; therefore, we determined whether HIF- $1 \alpha$ could also regulate these phenotypes in CMM cells. Transfection of WM451 and A375 cells with shLINC00518 reduced cell proliferation and increased apoptosis, while enhancing HIF-1 $\alpha$ in WM451 and A375 cells reversed this affect (Fig. 5A, B). Additionally, colony formation assays demonstrated that the cell survival capability of LINC00518-silenced cells was significantly decreased, while overexpression of HIF-1 $\alpha$ reversed this negative effect on cell survival (Fig. 5C). Wound-healing assays and Transwell Matrigel invasion assays suggested that LINC00518 is involved in regulating the migration and invasion in CMM cells, and that overexpression of HIF-1 $\alpha$ reversed LINC00518 knockdown-induced suppressing of migration, invasion in CMM cell lines (Fig. 5D, E). Our results show that LINC00518/HIF-1 $\alpha$ signaling loop participates in the regulation of proliferation, apoptosis, colony formation, migration, and invasion in CMM cells. 


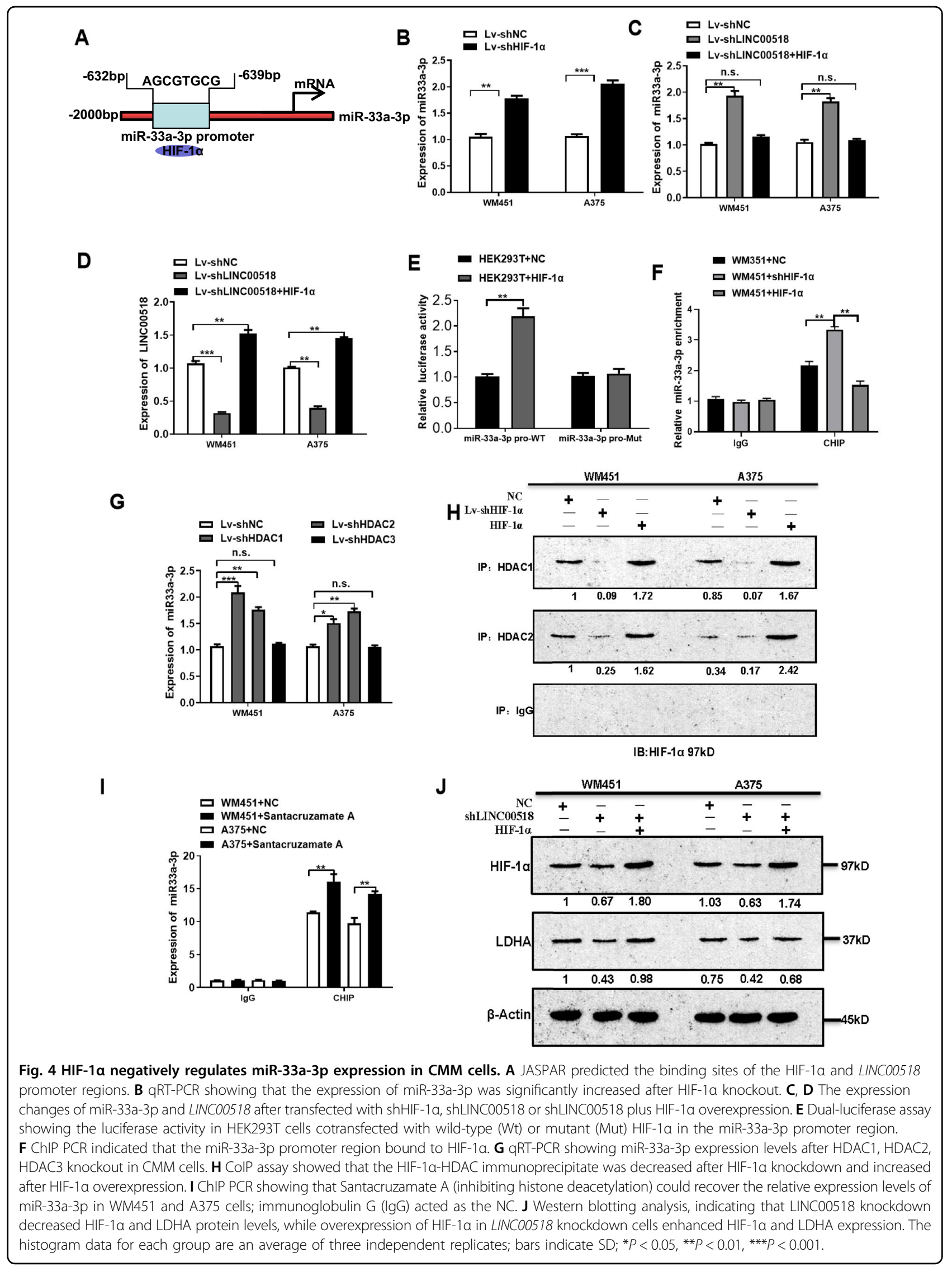




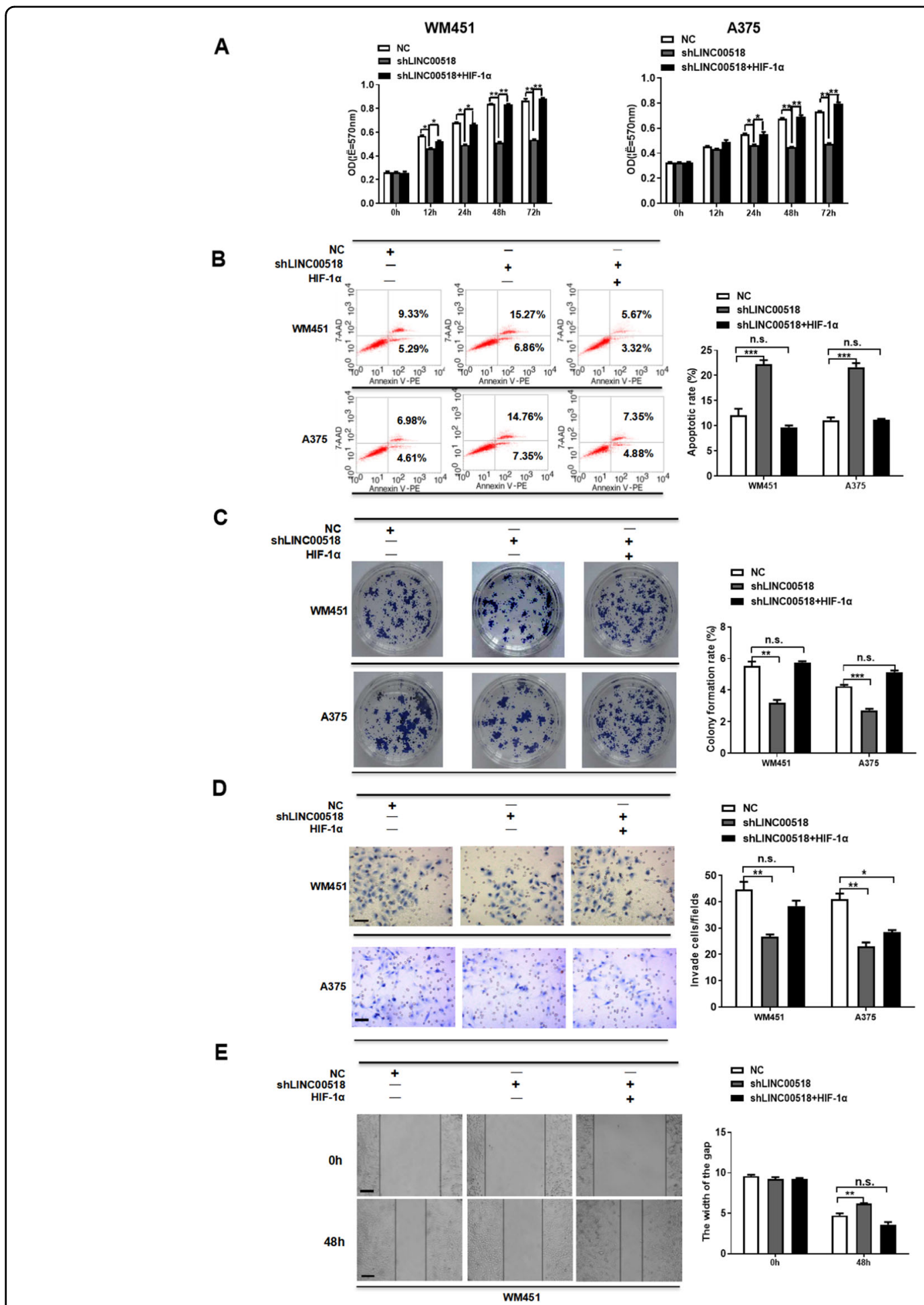

Fig. 5 Overexpression of HIF-1a reversed LINC00518 knockdown-induced suppressing of proliferation, migration, invasion, and colony formation. A WM451 and A375 cells were transfected with NC, shLINC00518, shLINC00518 plus HIF-1a overexpression plasmid; the MTT method was used to detect cell proliferation. B The effects of LINC00518 knockdown or LINC00518 knockdown plus HIF-1a overexpression on apoptosis of WM451 or A375 cells were assessed using annexin V staining and flow cytometry analysis. C Colony formation assay showed that LINC00518 silenced is involved in significantly decreasing cell proliferation, HIF-1a overexpression could reverse the regulatory effects of LINC00518. D Invasion of melanoma cells in different transfection groups was assessed by the transwell assay. E Wound-healing assay was used to assess the migration of melanoma cells in different transfection groups. The histogram data for each group are an average of three independent replicates; bars indicate SD; ${ }^{*} P<0.05,{ }^{* *} P<0.01,{ }^{* * *} P<0.001$ 
Involvement of the LINC00518/miR-33a-3p/HIF-1a negative feedback loop in glycolysis-mediated radiotherapy resistance of CMM cells

HIF- $1 \alpha$ has been reported to participate in regulating radiotherapy resistance by initiating glycolytic tumor metabolism in various cancers ${ }^{40}$. Therefore, we explored whether the LINC00518/HIF-1 $\alpha$ signaling loop affects the radiosensitivity of CMM. To further study the function of the LINC00518/HIF-1 $\alpha$ signaling loop in CMM cell radioresistance, CMM cells were treated with doses of $0-2,4$, and $6 \mathrm{~Gy}$, and MTT and Clonogenic assays were performed to determine their radiosensitivity. The results indicated that after exposure to a radiation dose of $2 \mathrm{~Gy}$, the proliferation and colony-forming capacity of CMM cell lines decreased significantly (Supplementary Fig. 1A,B). Furthermore, the protein levels of HIF- $1 \alpha$ and LDHA were increased in cells treated with 2 Gy of radiation (Supplementary Fig. 1C). These results indicated that treatment of CMM cells with 2 Gy of radiation could inhibit their cell proliferation and colony-forming abilities while maintaining their radioresistance. Therefore, we selected a single dose of 2 Gy as the radiation dose for subsequent experiments.

Western blotting confirmed that knockdown of LINC00518 expression in WM451 and A375 cells decreased HIF- $1 \alpha$ and LDHA protein levels, while overexpression of HIF- $1 \alpha$ could reverse this effect. To identify the role of the LINC00518/HIF-1 $\alpha$ signaling loop in mediating radiosensitivity of CMM cells, the effects of the glycolytic inhibitor, 2-deoxyglucose (2DG), and HDAC inhibitor, Santacruzamate A, were investigated in WM451 and A375 cells. Both 2DG and Santacruzamate A decreased HIF- $1 \alpha$ and LDHA protein levels, which were inhibited by HIF-1 $\alpha$ overexpression or by upregulation of LINC00518 and HIF-1 $\alpha$ in CMM cells (Supplementary Fig. 1D).

To determine whether LINC00518/HIF-1 $\alpha$ plays a role in anaerobic glycolysis of CMM, we transfected WM451 and A375 cells with a LINC00518 expression-silencing plasmid or a LINC00518 expression-silencing plasmid together with an HIF-1 $\alpha$-overexpression plasmid, and biochemically examined glucose consumption, ATP levels, and lactic acid production. Knockdown of LINC00518 expression in WM451 and A375 cells decreased glucose consumption, ATP levels, and lactic acid production in the cells, while overexpression of HIF- $1 \alpha$ significantly reversed the effects of LINC00518 silencing of glycolytic tumor metabolism in WM451 and A375 cells (Fig. 6A-C). These results suggest that the LINCO0518/HIF-1 $\alpha$ signaling loop could increase CMM cell glycolytic metabolism.

MTT, flow cytometry, colony formation, and immunofluorescence assays revealed that knockdown of LINC00518 expression in WM451 and A375 cells increased radiosensitivity, while overexpression of HIF- $1 \alpha$ reversed this effect. Both 2DG and Santacruzamate A increased radiosensitivity, which was inhibited by HIF- $1 \alpha$ overexpression or by upregulation of LINCO0518 and HIF-1 $\alpha$ in CMM cells (Figs. 6D, E and 7A, B). The results demonstrated that the LINC00518/HIF-1 $\alpha$ signaling loop could regulate the HIF$1 \alpha$-LDHA pathway and promote glycolytic metabolism, thereby increasing the radioresistance of CMM cells.

\section{Effect of the LINC00518/miR-33a-3p/HIF-1 a negative feedback loop on radiosensitivity in CMM cells in vivo}

We used a xenograft mouse model to confirm the effect of LINC00518/HIF-1 $\alpha$ on the radiosensitivity of CMM cells in vivo. Repression of LINC00518 expression using an shLINC00518 plasmid, or Santacruzamate A, reduced the tumorigenic ability of WM451 and A375 cells, and increased their radiosensitivity in the subcutaneous sarcoma model (Fig. 8A).

To analyze the relationship between radiosensitivity and glycolysis, immunohistochemistry and western blotting analysis were conducted to measure HIF- $1 \alpha$ and LDHA protein levels in the xenograft tumors. Immunohistochemistry showed that HIF-1 $\alpha$, Ki67, and LDHA proteins were significantly downregulated following knockdown of LINC00518 in WM451 and A375 cells or in cells treated with Santacruzamate A (Fig. 8B). Western blotting also confirmed the above-mentioned results (Fig. 8C). The subcutaneous sarcoma model further confirmed that the LINC00518/miR-33a-3p/HIF-1 $\alpha$ negative feedback loop could increase radiotherapy resistance, accompanied by accelerated glycolysis. A schematic of this search is shown in Fig. 8D.

\section{Discussion}

The long intergenic nonprotein coding RNA 518 (LINC00518) is located on human chromosome 6p24.3 and shows low expression in most normal and malignant human tissues. However, its expression is markedly upregulated in some tumor tissues ${ }^{33,36,37,41}$. LINC00518 has been shown to contribute to chemoresistance and promote epithelial cell growth through regulating the miR-199a/MRP1 signaling in breast Cancer ${ }^{36}$. Knockdown of LINC00518 inhibits cervical cancer proliferation invasion and migration by regulating JAK/STAT3 axis ${ }^{37}$. Some studies have reported that LINC00518 is highly expressed in melanoma and plays a key role in the malignant progression of melanoma ${ }^{33,42}$. The above data indicate that LINC00518 may be an important cancer gene, and may be involved in the occurrence and development of tumors through various mechanisms. However, the role of LINC00518 in the process of CMM radioresistance has not yet been studied.

LncRNAs play an important role in the complex etiology and mechanism of carcinogenesis progression ${ }^{43,44}$. In the present study, we noted that LINC00518 expression was significantly upregulated in CMM tissues compared with that in normal skin tissues, and that patients with 
A

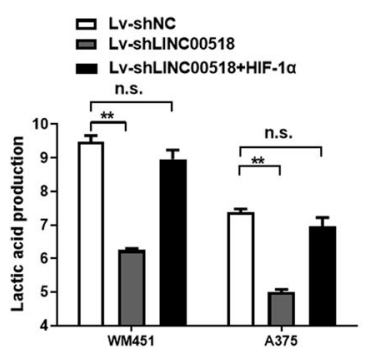

D
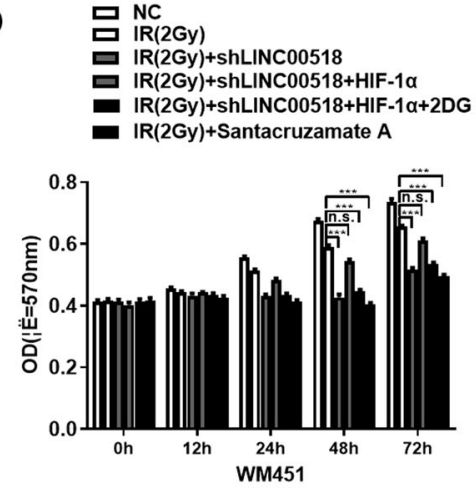

B 믄.shNC

C. LV-ShLLINC00518+HIF-1a

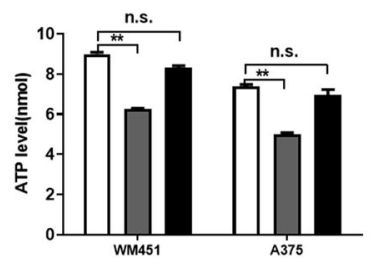

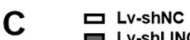

- Lv-shLINC00518+HIF-1a

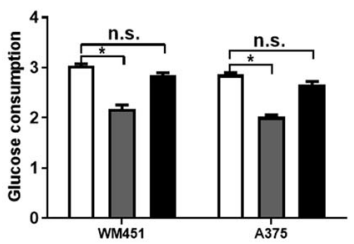

E

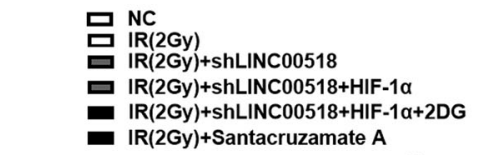

므수 $\mathrm{NC}$

므 IR(2Gy)+shLINC00518

口 IR(2Gy)+shLINC00518+HIF-1

- IR(2Gy)+Santacruzamate A
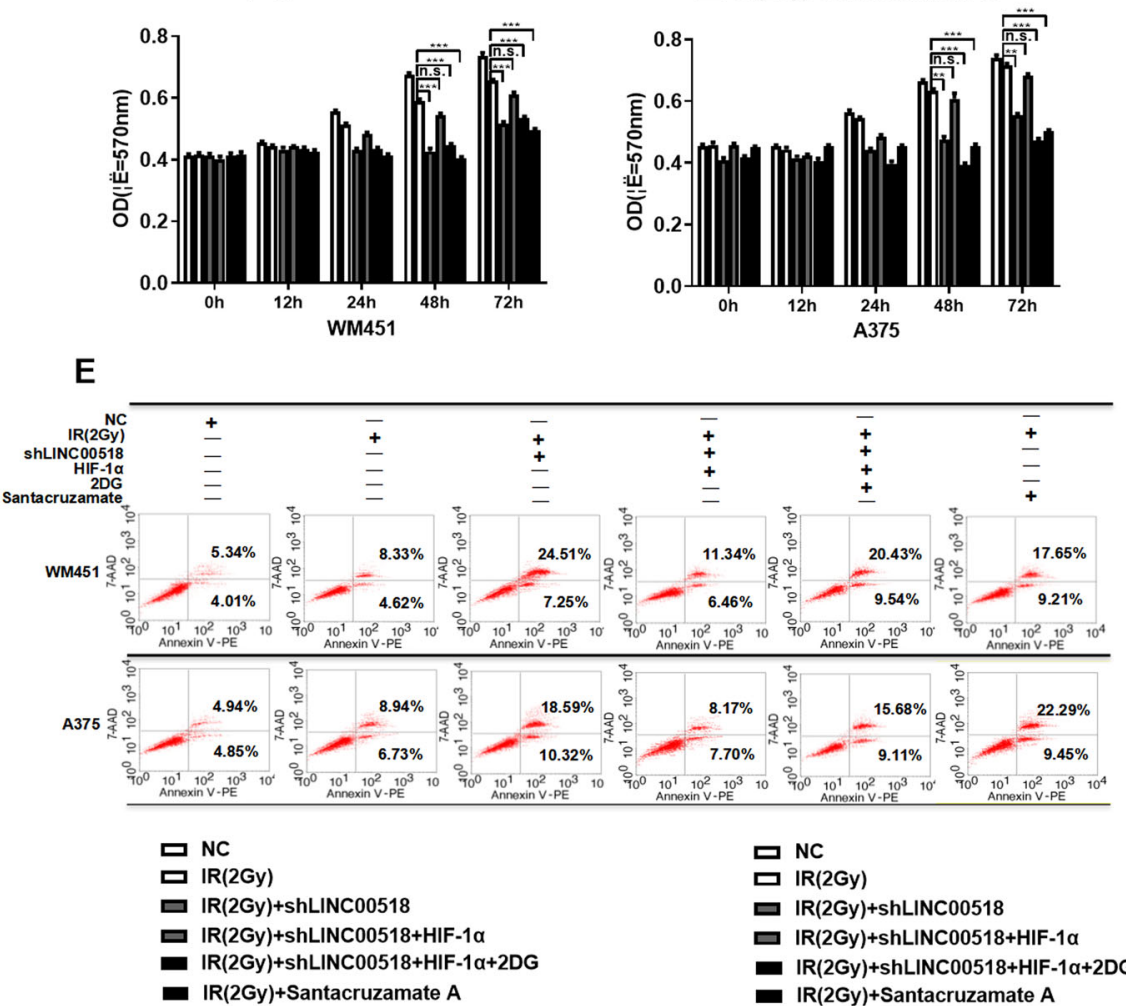

口 NC

口 IR(2Gy)

口 IR(2Gy)+shLINC00518

口 IR(2Gy)+shLINC00518+HIF-1

- IR(2Gy)+shLINC00518+HIF-1a+2DG

- $\operatorname{IR}(2 G y)+$ Santacruzamate $A$
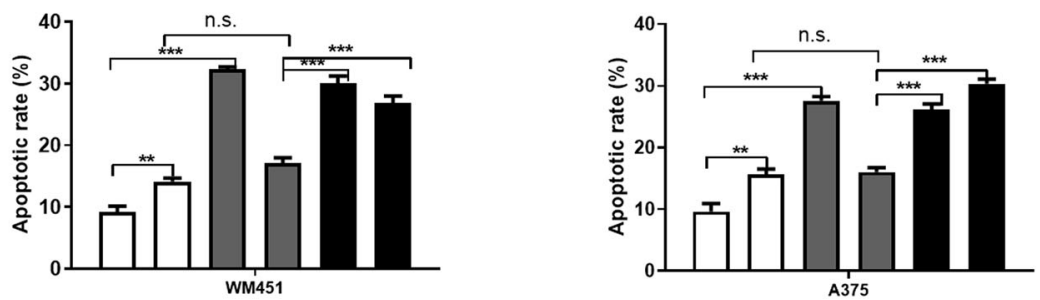

Fig. 6 Involvement of the LINC00518-miR-33a-3p-HIF-1a negative feedback loop in glycolysis-mediated radiotherapy resistance of CMM cells. A-C The levels of glucose consumption and lactate production were estimated in CMM cells. These results indicated that knockdown of LINC00518 expression in WM451 and A375 cells decreased glucose consumption, ATP levels, and lactic acid production in the cells; by contrast, overexpression of HIF-1a significantly reversed the effects of LINC00518 silencing on glycolytic tumor metabolism in WM451 and A375 cells.

D Detection of CMM cell sensitivity to radiotherapy after LINC00518 knockdown by using an MTT assay. LINC00518 knockdown in CMM cells could inhibit their proliferation after the use of radiotherapy and HIF-1a could reverse this effect, while 2DG and Santacruzamate A offset the influence of HIF1a. E Flow cytometry examination of the effect of radiotherapy on CMM cell apoptosis following LINC00518 knockdown. LINC00518 knockdown in CMM cells could increase apoptosis after using radiotherapy and HIF-1a could reverse this effect, while 2DG and Santacruzamate A offset the influence of HIF-1a. The histogram data for each group are an average of three independent replicates; bars indicate SD; ${ }^{*} P<0.05,{ }^{* *} P<0.01,{ }^{* * *} P<0.001$. 


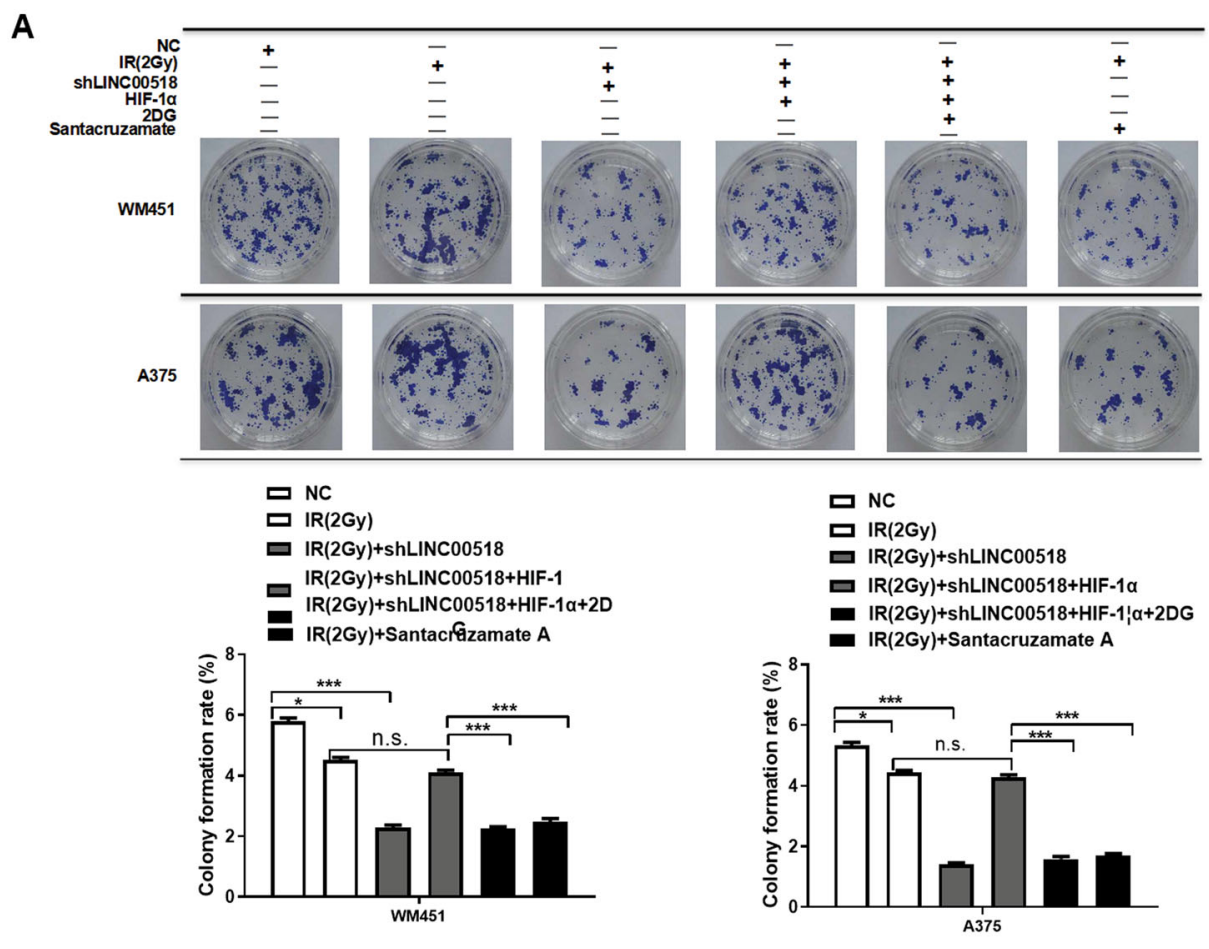

B

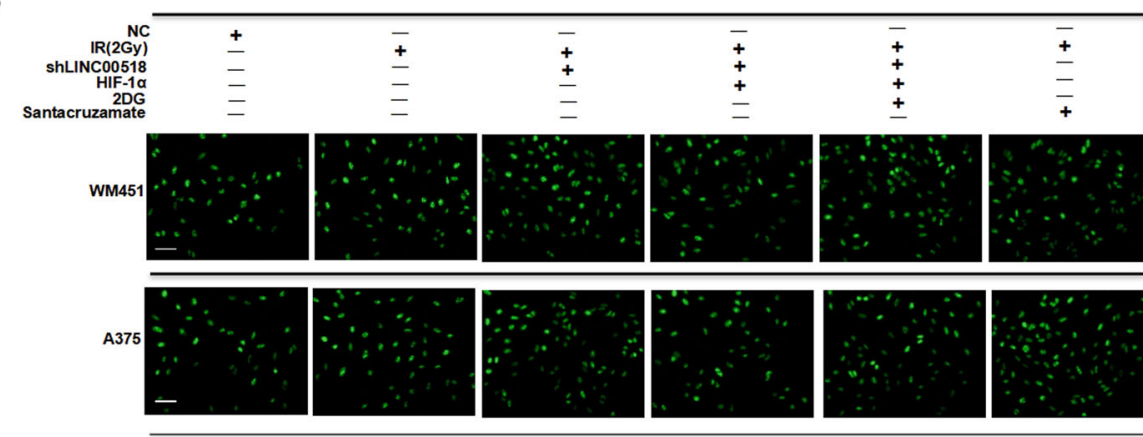

口C

口 IR(2Gy)

口 IR(2Gy)+shLINC000518

口 IR(2Gy)+shLINC000518+HIF-1a

- IR(2Gy)+shLINC000518+HIF-1 $\alpha+2 D G$

- IR(2Gy)+Santacruzamate A

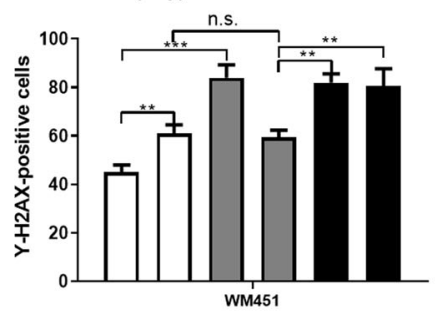

口C

IR(2Gy)

口 IR(2Gy)+shLINC000518

口 IR(2Gy)+shLINC000518+HIF-1 $\alpha$

- IR(2Gy)+shLINC000518+HIF-1a+2DG

- IR(2Gy)+Santacruzamate A

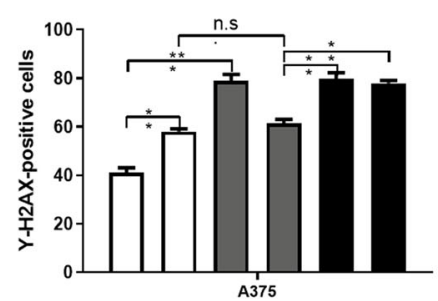

Fig. 7 Involvement of the LINC00518/miR-33a-3p/HIF-1a negative feedback loop in glycolysis-mediated radiotherapy resistance of CMM cells. A Colony formation showing that knockdown of LINC00518 expression in WM451 and A375 cells inhibited the invasion and proliferation of CMM cells, increased the radiosensitivity of cells, while overexpression of HIF-1a reversed this effect. Both 2DG and Santacruzamate A increased radiosensitivity, which was inhibited by HIF-1a overexpression or by upregulation of LINC00518 and HIF-1a in the CMM cells. B Immunofluorescence assays indicating that knockdown of LINC00518 expression in WM451 and A375 cells could induce structural damage of their nuclei under radiotherapy; however, HIF-1a could reverse this effect, while 2DG and Santacruzamate A offset the influence of HIF-1a. The histogram data for each group are an average of three independent replicates; bars indicate SD; ${ }^{*} P<0.05,{ }^{* *} P<0.01,{ }^{* * *} P<0.001$. 

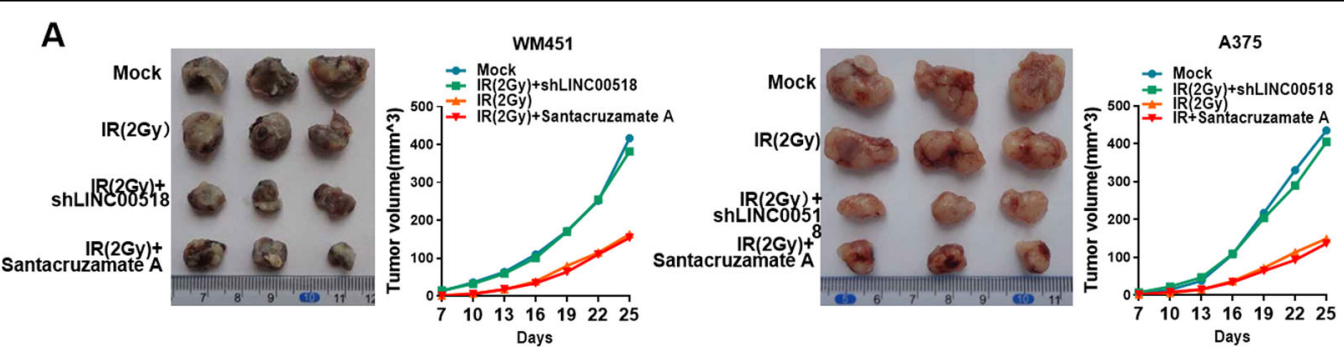

\section{B}
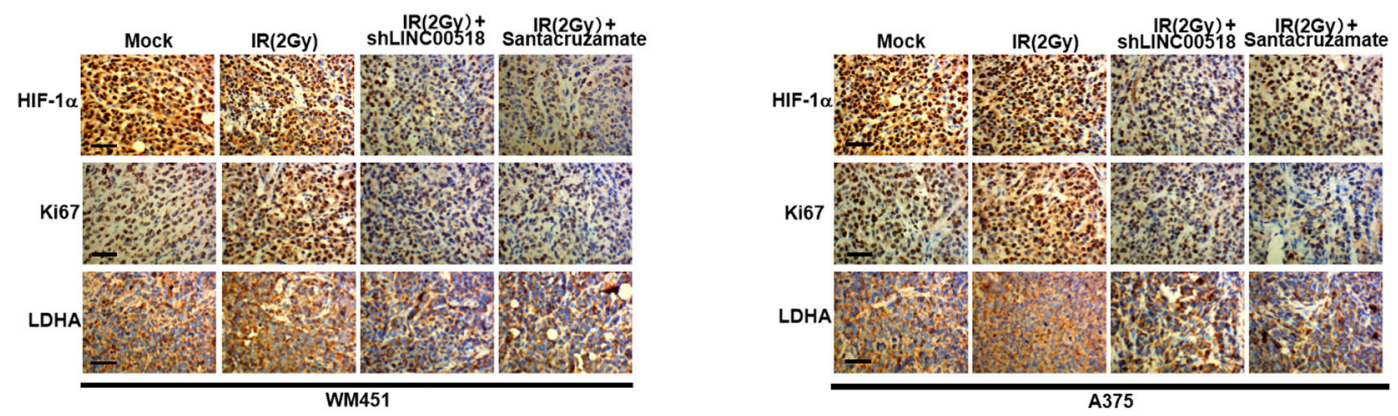

C
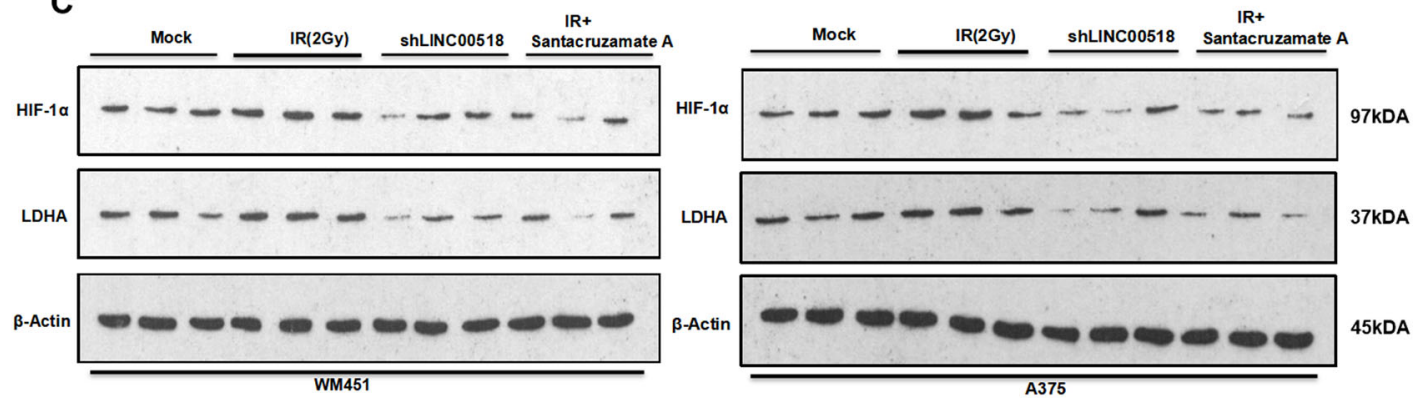

D

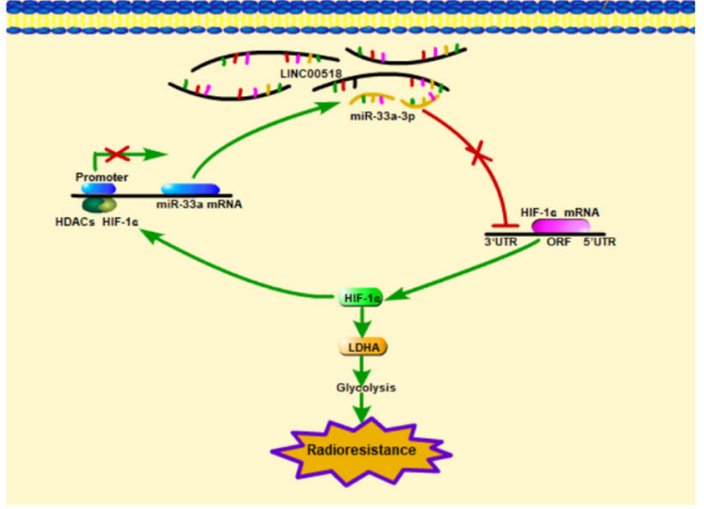

Fig. 8 Effect of the LINC00518/miR-33a-3p/HIF-1a negative feedback loop on radiosensitivity in CMM cells in vivo. A Transplantation tumor experiments showing that repression of LINC00518 expression using the shLINC00518 plasmid or treating the cells with Santacruzamate A reduced the tumorigenic ability and tumor volume of WM451 and A375 cells, thereby increasing their radiosensitivity as observed by reduced colony formation in the subcutaneous sarcoma model. B Immunohistochemical assays showing that knockdown of LINC00518 expression or treating the cells with Santacruzamate A decreased HIF-1a, Ki67, and LDHA protein levels in tumor tissues of mice. C Western blotting showing that LINC00518 expression or treatment of the cells with Santacruzamate A decreased HIF-1a and LDHA protein expression in tumor tissues of mice. D Schematic of this search. The histogram data for each group are an average of three independent replicates; bars indicate SD; ${ }^{*} P<0.05,{ }^{* *} P<0.01,{ }^{* * *} P<0.001$. 
CMM with high expression of LINC00518 had a remarkably poor prognosis in terms of both RFS and OS. This indicates that LINCO0518 may be a molecular target in CMM cells and a potential biomarker for the diagnosis and prognosis of patients with CMM. Additionally, LINC00518 was shown to increase the proliferation and clonogenicity of CMM cells, induce their migration and invasion, and decrease their apoptosis, suggesting that LINC00518 acts as an oncogene in CMM cells.

LINC00518 primarily localized in the cytoplasm, regulates the target genes of miRNAs by acting as miRNA sponges, thereby inhibiting their functions ${ }^{36,37}$. Therefore, we identified the miRNAs that bind to LINC00518. Further studies demonstrated that LINCO0518 directly targets miR33a-3p, which, in turns, targets HIF-1 $\alpha$. LINC00518 upregulates HIF- $1 \alpha$ expression by binding competitively to miR-33a-3p. Previous studies have shown that transcription factors could promote lncRNA expression by binding to the promoter region of the gene ${ }^{45-47}$; however, the influence of transcription factors on the regulation of miRNA expression is rarely reported. Our study is the first to demonstrate that HIF- $1 \alpha$ could bind to the miR-33a-3p promoter region and recruit HDAC in CMM cells and restrain miR-33a-3p expression by promoting miR-33a histone deacetylation. HDACs deacetylate histones and nonhistone proteins, which influence regulation of gene transcriptio $^{48}$. In cancer cells, inhibition of HDACs has been shown to influence the execution of extrinsic and intrinsic apoptosis pathways as well as DNA repair ${ }^{49-51}$. Our results also provide a new aspect and method to explore the interaction between histone deacetylation and transcription factors. Besides, LINC00518, miR-33a-3p, and HIF- $1 \alpha$ could comprise a negative feedback loop, providing the first evidence for the presence of a LINC00518-miR$33 a-3 p-H I F-1 \alpha$ regulatory axis in CMM cells.

Next, we verified whether LINCO0518 and HIF- $1 \alpha$ could specifically inhibit miR-33a-3p expression. MiR-33a-3p suppresses a malignant phenotype by inhibiting the expression of pre-B-cell leukemia homeobox in hepatocellular cancer ${ }^{52}$, and increased miR-33a-3p expression can promote the malignant phenotype of gastric carcinoma ${ }^{53}$. Metastasis is a multistep process that includes de-adhesion, migration, adhesion, and invasion; however, the influence of miR-33a-3p on the CMM phenotype has not been studied. To investigate the role of miR-33a-3p in CMM in vitro, malignant phenotype assays were performed after overexpressing miR-33a-3p in CMM cells. We found that miR33a-3p acts as an antioncogene by inhibiting the activity of HIF- $1 \alpha$ in CMM. Our findings indicated that miR-33a-3p might be a tumor suppressor, which has an enormous value in diagnostic and prognostic evaluation and in the development of new targeted therapy of CMM.

Glycolysis supports the uninterrupted growth of cancer cells, while higher glycolytic rate in tumor cells is considered the primary cause of radiotherapy failure due to radioresistance ${ }^{54,55}$. Thus, interrupting or disrupting tumor glycolysis will impact tumor growth via energy depletion as well as sensitization to therapeutics. Hypoxia and nutrient deficiency are common phenomena in advanced solid human tumors. As a hypoxia-inducible factor, HIF- $1 \alpha$ can be stimulated by these stress conditions ${ }^{56,57}$. HIF- $1 \alpha$ activation in tumor cells is one of the key masters orchestrating their adaptation mechanisms to the hypoxia environment. However, in a separate study, a significant correlation has not been established between HIF-1 $\alpha$ and the prognosis of malignant melanoma, their results indicated that HIF- $1 \alpha$ overexpression is present in most primary melanomas, but is not associated with clinicopathological variables, patient prognosis, or survival ${ }^{58}$. Our study found that the HIF-1 $\alpha$ LDHA pathway regulates the activation of anaerobic glycolysis in tumor cells and suppresses cells that are sensitive to radiotherapy and chemotherapy.

Biochemical tests of glucose consumption, ATP levels, and lactic acid production suggested that the LINC00518-miR$33 a-3 p-H I F-1 \alpha$ negative feedback loop plays a major role in glycolysis of CMM cells. Thus, we hypothesized that this negative feedback loop might inhibit the radiotherapy sensitivity of CMM cells. To test this hypothesis, in vitro radio response assays were conducted following LINC00518 knockdown of LINCO0518 in CMM cells. Considering that the expression of LINCO0518 was very high in these cells, overexpression of LINC00518 was not evaluated. We found that the LINCO0518-miR-33a-3p-HIF-1 $\alpha$ negative feedback loop could increase radiation resistance in CMM by regulating the HIF- $1 \alpha$-LDHA glycolysis signaling pathway. Therefore, anticancer therapies that target the LINC00518-miR-33a-3p-HIF-1 $\alpha$ negative feedback loop might be an effective therapeutic strategy to enhance CMM cell radiosensitivity.

In conclusion, LINC00518 is highly expressed in CMM tissues, and high expression of LINC00518 is an indicator of poor prognosis in patients with CMM. The LINC00518-HIF- $1 \alpha$-glycolysis axis can promote oncogenesis and the development of CMM cells by allowing CMM cells to adapt to a state of tumor hypoxia. The LINC00518-HIF-1 $\alpha$-glycolysis axis can also significantly upregulate HIF-1 $\alpha$ and LDHA, thereby inhibiting apoptosis and inducing proliferation of CMM cells in response to radiation, ultimately causing radioresistance. Our results demonstrated that LINC00518 might be a prognostic biomarker and an actionable target for radiosensitization of CMM.

\section{Materials and methods \\ Data mining and analysis}

To identify functional lncRNAs in CMM gene expression patterns of two different cohorts of CMM, derived from the Affymetrix GeneChip ${ }^{\circledR}$ Human Genome U133 
Plus 2.0 Array, GSE46517 and GSE4587 were downloaded from the GEO database (http://www.ncbi.nlm.nih.gov/ geo/). These two cohorts were based on the same platform of array chip and performed whole-genome expression profiling of human tissue specimens representing normal skin and advanced-stage melanomas. In addition, original data of these two cohorts were accessible. The Significant Analysis of Microarray (SAM) mode was used to analyze the different expression levels of lnRNAs between adjacent and CMM tissue samples in these two data sets. The critical value for the expressed lncRNAs was set at $\geq 2.0$-fold change and a false discovery ratio of $<0.05$.

\section{Cell lines and tissue}

Human melanoma cell lines (WM35, WM451, and A375) and HM were purchased from the ATCC (Rockville, MD, USA) and cultured in Dulbecco's modified Eagle's medium supplemented with $10 \%$ fetal bovine serum (Gibco, Carlsbad, USA) and $1 \%$ penicillin/streptomycin under a humidified atmosphere of $5 \% \mathrm{CO}_{2}$ at $37^{\circ} \mathrm{C}$. Human CMM tissues and corresponding normal skin tissues were collected from patients who underwent surgery and were pathologically diagnosed with MM at the Third Xiangya Hospital of the Central South University and Hunan Cancer Hospital from January 2015 to December 2017. Informed consent was obtained from all the participants.

\section{Cell transfection}

MiR-33a-3p mimics and inhibitors, LINC00518shRNAs, HIF_1 $\alpha$-shRNAs, HIF- $1 \alpha$ overexpression plasmid, and negative controls (NC) were purchased from GenePharma (GeneChen, Shanghai, China). The LINC00518-shRNAs we used have been validated in the previous publication ${ }^{33}$. Cells were transfected as previously described ${ }^{59}$. CMM cells were cultured in six-well plates and allowed to reach $70 \%$ confluence after $24 \mathrm{~h}$. Lipofectamine $^{\mathrm{TM}} 2000$ (Invitrogen, Carlsbad, USA) was used to transfect cells with DNA complexes following the manufacturer's instructions. The cells were harvested, transfected, and RNA and protein were extracted after 24 and $36 \mathrm{~h}$, respectively. Real-time PCR was used to confirm the efficiency of transfection.

The target sequences of LINC00518 shRNA were: GGACATTTCCTGTCTGCAATT.

\section{Measurement of glucose consumption and lactate production}

Glucose consumption and lactate production were estimated as previously described ${ }^{60}$. Briefly, cells $\left(5 \times 10^{5}\right)$ were recovered and cultured in six-well plates. Following incubation for $10 \mathrm{~h}$, the cell culture medium was removed and the cells further incubated with fresh medium for $8 \mathrm{~h}$. Glucose and lactate levels were measured using
Automatic Biochemical Analyzer, 7170A (HITACHI, Tokyo, Japan) at the Clinical Biochemical Laboratory of Third Xiangya Hospital. The relative rates of glucose consumption and lactate production were normalized using consistent amounts of protein.

\section{Quantitative real-time reverse transcription PCR}

Total RNA was extracted from the cells using TRIzol reagent (Invitrogen, Carlsbad, USA). The PARIS kit (Ambion, Foster City, USA) was used to isolate RNA from the cytoplasm and nuclei of transfected cells. Real-time PCR was performed using RNA isolated from the cytoplasmic and nucleic fractions. RNA purity was assessed by spectrophotometry (A260/A280 > 1.8). To perform qRTPCR, $1 \mu \mathrm{g}$ of RNA from the samples was reverse transcribed using a RevertAid ${ }^{\mathrm{TM}} \mathrm{H}$ Minus First Strand cDNA Synthesis Kit (Fermentas \#K1631; Thermo Fisher Scientific, Waltham, MA, USA). qRT-PCR was performed to assess the expression of LINC00518 and miR-33a-3p using a SYBR Green PCR Master Mix (Toyobo, Shanghai, China) employing the CFX96 Real-Time PCR Detection System (Ambion, Foster City, USA). PCR amplifications were performed in triplicates. The sequences of the qRTPCR primers were as follows:

\section{LINC00518: forward: 5'-TGCAATTCAGGTCGGTTG TA-3'; \\ reverse: $5^{\prime}$-GTGGAGCTCCCTGAAGACAG- $3^{\prime}$. miR-33a-3p: forward: 5'-ACACTCCAGCTGGGCAAT GTTTCCACAGTG-3'; \\ reverse: 5'-CTCAACTGGTGTCGTGGAGTCGGCAA TTCAGTTGAGGTGATGCA- $3^{\prime}$. \\ $\beta$-actin: forward: 5'-CATGTACGTTGCTATCCAGGC-3'; reverse: 5'-CTCCTTAATGTCACGCACGAT-3'.}

$A C T B$ (encoding $\beta$-actin) was used as an internal reference.

\section{Western blotting}

Phosphate-buffered saline (PBS) was used to wash the cancer biopsy specimens and cancer cells, and later the cells were lysed on ice using the radioimmunoprecipitation assay (RIPA) buffer containing $10 \%$ protease inhibitor cocktail (Roche Applied Science, Basel, Switzerland) for $30 \mathrm{~min}$. Proteins were quantified using a Total Protein Extraction Kit (ProMab, Richmond, CA, USA). The proteins were separated using a 10\% SDS-polyacrylamide gel and transferred to polyvinylidene fluoride membranes. The membranes were incubated with the following primary antibodies: anti-HIF- $1 \alpha$ (1:1000, Bioss, bs-20398R), anti-LDHA (1:50, Proteintech, 19987-1-AP), anti-Histone H3 (acetyl K4) (1:500, Abcam, ab232931), anti-Histone H3 (acetyl K9) (1:2000, Abcam, ab4441), anti-Histone H3 (acetyl K27) (1:200, Abcam, ab177178), anti-Histone H3 
(1:1000, CST, \#4499), and anti- $\beta$-Actin (1:2000, CST, \#3700S) at $4{ }^{\circ} \mathrm{C}$ overnight, followed by the addition of HRP-conjugated secondary antibodies at $37{ }^{\circ} \mathrm{C}$ for $60 \mathrm{~min}$. The signals of the immunoreactive protein bands were assessed using densitometric analysis software and quantified by densitometry using the ImageJ software $(\mathrm{NIH}$, Washington, USA). $\beta$-actin served as a loading control.

\section{Invasion and migration assays}

The migration capacity of CMM cells was assessed using a wound-healing assay. Cells $\left(1 \times 10^{5}\right.$ per ml $)$ were cultivated in six-well plates and grown to $85-90 \%$ confluence. A wound was scratched in the middle of the monolayer using a $10-\mu \mathrm{L}$ pipette tip, and the cells were washed twice with PBS to dislodge cellular debris, after creating the wound, the $10 \%$ FBS-containing medium was replaced with medium containing 1\% FBS concentration. A photograph was taken at $0 \mathrm{~h}$ under a microscope. The plates were placed in a humidified atmosphere of $5 \% \mathrm{CO}_{2}$ at $37^{\circ} \mathrm{C}$, and the wound was photographed again at $48 \mathrm{~h}$. The difference between the two measured widths on the images represented the extent of cell migration.

CMM cell invasion capacity was detected using a Transwell assay. Cells $\left(1 \times 10^{5}\right)$ in $100 \mu \mathrm{L}$ of serum-free medium were added to the apical chamber of a Transwell inserts ( $8 \mu \mathrm{m}$ pore size, BD Biosciences, New Jersey, USA) in 24well plates. Transwell inserts were pre-coated with Matrigel matrix of $300 \mu \mathrm{g} / \mathrm{ml}$ (Biosciences, New Jersey, USA), and $500 \mu \mathrm{L}$ of $10 \%$ FBS-containing medium was added to the lower chamber. After incubation for $48 \mathrm{~h}$ at $37^{\circ} \mathrm{C}$, the invading cells that traversed the membrane were fixed with methanol and stained with $0.1 \%$ crystal violet. Cells that remained on the upper chamber surface of the Transwell membrane were wiped off using a cotton bud. Six visual fields at $\times 20$ magnification were randomly captured and the numbers of invaded tumor cells were counted.

\section{Cell proliferation bioassay}

CMM cells treated with a short interfering RNA (siRNA) or plasmid were cultivated in 96-well plates (500 cells per well). Cell proliferation after irradiation or transfection was determined using a commercial MTT assay kit (Sigma Aldrich, St. Louis, MO, USA) according to the manufacturer's instructions. First, $50 \mu \mathrm{L}$ of MTT was added to the cells in the wells and incubated for $4 \mathrm{~h}$. Later, $150 \mu \mathrm{L}$ of dimethyl sulfoxide was added to dissolve the MTT crystals. The light absorption value of each well was measured using a spectrometer at $570 \mathrm{~nm}$. All the assays were repeated no less than three times in triplicate wells.

\section{Cell cycle and apoptosis assays}

Cell cycle and apoptosis assays were performed using flow cytometry. CMM cells transfected with siRNA or vector were cultivated in six-well plates. Cells were treated with or without irradiation for $48 \mathrm{~h}$, and then harvested by centrifugation. An annexin V-fluorescein isothiocyanate/ propidium iodide staining kit (MB-CHEM Mumbai, India) and cell cycle detection kit (Sigma) were used to stain the cells, according to the manufacturers' protocols. All the tests were performed in triplicates.

\section{Clonogenic assay}

A clone formation assay was used to assess the proliferation activity and cellular radiosensitivity of $\mathrm{MM}$ cells $^{61}$. X-ray irradiation was performed using an X-ray generator (Varian, California, USA), emitting at a fixed dose rate of $4 \mathrm{~Gy} / \mathrm{min}$. The energy of the X-rays used to irradiate the cells was graded as 0-2, 4, 6 Gy. Cells transfected with the siRNA or plasmid were seeded in six-well plates at 1000 cells per well. The cells were treated with or without irradiation or additional processing and then incubated for 14 days to form colonies. The culture medium was replaced at intervals of 2-3 days. MM cells were washed twice with PBS before being harvested and colonies are fixed with glutaraldehyde $(6.0 \% \mathrm{v} / \mathrm{v})$ for $15 \mathrm{~min}$, stained with hematoxylin $(0.5 \% \mathrm{w} / \mathrm{v})$ for $30 \mathrm{~min}$. The surviving fractions ( $>50$ cells) were counted under a microscope. All the clone formation assays were performed in triplicates.

\section{Dual-luciferase experiment}

For the luciferase reporter experiment, the LINCO05 18-WT, LINC00518-MUT, miR-33a-3p-pro-WT, miR33a-3p-pro-MUT, HIF-1 $\alpha-3^{\prime}-\mathrm{UTR}-\mathrm{WT}$, and HIF- $1 \alpha-3^{\prime}-$ UTR-MUT vectors were used. WM451 and A375 cells were seeded in six-well plates and transfected with hsamiR-33a-3p mimics or empty plasmids for $48 \mathrm{~h}$. The dualluciferase reporter system (Glomax, Promega, Madison, USA) was used to assess firefly and sea pansy luciferase activities. The relative luciferase activities were calculated, and control cells were used for normalization.

\section{Chromatin immunoprecipitation (ChIP)}

WM451 and A375 cells were treated with or without Santacruzamate A (an HDAC inhibitor) and underwent two rounds of dual crosslinking. The protein-DNA complexes were precipitated using anti-H3K27AC antibodies (ab177178). Signal intensity of the promoter region of the target gene was determined from PCR results. The signal strength of the miR-33a-3p promoter was measured by PCR using a SYBR Green PCR Master Mix Kit (ABI 4309155). According to the promoter region of miR-33a$3 p$, containing HIF-1 $\alpha$-binding sites (AGACGTGA and GGGCGTGG), primers were designed to analyze the purified DNA. The following primer sequences for qRTPCR were used:

miR-33a-3p: forward: 5'-CTTAGCAGCAGACGTGAT GG-3'; 
reverse: 5'-GAGTCGAGAGGCAGGTCACT-3'. GAPDH: forward: 5'-GTCAACGGATTTGGTCTGTA TT-3'; reverse: 5'-AGTCTTCTGGGTGGCAGTGAT-3' .

\section{RNA pull-down assay}

An RNA pull-down assay was performed using the Magnetic RNA-Protein Pull-Down Kit $\left(\right.$ Pierce $^{\mathrm{TM}}$, Thermo, Shanghai, China). In vitro biotin-labeled (Bio)-miR-NC, Bio-miR-33a-3p, and Bio-miR-33a-3p-Mut were transfected into WM451 and A375 cells, and the cells were cultured for $48 \mathrm{~h}$. The cell lysates were collected and incubated with streptavidin magnetic beads, forming protein-Bio/RNA-magnetic bead complexes. High salt elution was used to obtain the protein-bio/RNA from the magnetic bead-protein-bio/RNA mixture. The TRIzol reagent (Invitrogen, Carlsbad, USA) was used to purify the protein-bio/RNA, and qPCR was used to measure the relative expression of LINCO0518.

\section{Co-immunoprecipitation assay}

WM451 and A375 cells transfected with an siRNA or a plasmid overexpressing HIF- $1 \alpha$ were seeded in a $10 \mathrm{~cm}$ petri dish. The cells were washed twice with pre-chilled PBS and disrupted using RIPA lysis buffer $\left(1 \mathrm{~mL}\right.$ for $10^{7}$ cells). Protein A + G Agarose (Bioss, P1012) was used to precipitate protein complexes. The collected complexes were subjected to SDS-PAGE followed by western blotting. Anti-HDAC1 (1:100, SAB, 32034), anti-HDAC2 (1:100, Abcam, ab12169), and anti-HIF-1 $\alpha$ (1:200, Bioss, bs-20398R) were used as detection antibodies; anti-IgG (1:150, Bioss, bs-0297P) was used as a loading control.

\section{Xenograft mouse model}

Male BALB/c nude mice (5 weeks old, $18 \pm 0.75 \mathrm{~g}$ ) were purchased from the Laboratory Animal Center of Central South University (Changsha, China) and were maintained under specific pathogen-free conditions (IVC) in the Experimental Animal-feeding Centre of the Xiangya Medical College of Central South University (Changsha, China). To generate tumors in vivo, WM451 and A375 cells $\left(1 \times 10^{6}, 0.2 \mathrm{ml}\right)$ were treated with shRNA-mock1, shRNA-mock2, shRNA-LINC00518transfected, or Santacruzamate A, co-cultured, and injected into mice that were divided into four groups: A-D ( $n=3$ animals per group, 5 weeks old); knockdown assays were conducted as previously described ${ }^{32}$. Cells were injected subcutaneously on the superior border of the right upper limb, and isoflurane anesthesia machine was used to administer inhalational anesthesia to mice. Induction: $2-4 \%$, ZS, Beijing, ZS-MV-HR. When the mice developed a $100-\mathrm{mm}^{3}$ tumor a single 2 Gy dose of irradiation was delivered to the B-D groups. The tumor volume was estimated every 2 days by measuring the tumor length (L) and width (W). The tumor volume (V) was calculated using the formula $V=1 / 2\left(L \times W^{2}\right)$. After 40 days, mice were sacrificed by cervical dislocation at the Laboratory Animal Center of the Xiangya Medical College of Central South University, and the tumors were collected. All animal experiments were approved by the Ethics Committee of the Third Xiangya Hospital of Central South University.

\section{Statistical analysis}

The statistical software package GraphPad Prism version 5.0 (GraphPad Software, Inc., La Jolla, CA, USA) was used for all statistical analyses. All experiments were conducted at least in triplicates. All data are shown as mean \pm SD, and $P<0.05$ was considered statistically significant. Differences between the groups were analyzed using analysis of variance followed by comparison between specific groups using Student's $t$ test.

\section{Acknowledgements}

The authors thank Dr. J.Z. for her helpful suggestions to improve this paper.

\section{Author details}

'Department of Oncology, Third Xiangya Hospital, Central South University, Changsha, 410013 Hunan, PR China. ${ }^{2}$ Department of Plastic Surgery, Third Xiangya Hospital, Central South University, Changsha, 410013, PR China. ${ }^{3}$ Department of Respiration, the Second People's Hospital of Hunan Province of Hunan University of Chinese Medicine, Changsha, 410000, PR China

\section{Author contributions}

Y.L. and K.C. designed the study, analyzed and interpreted the data, and wrote the paper. D.H. contributes to data acquisition and interpretation. Y.L. and M.X performed all bioinformatics analysis and carried out the experiments. Y.Z. and J.Z. provide technical expertise and support. All authors have seen and approved the final version of the paper.

\section{Funding}

This work was supported by National Natural Science Foundation of China (81874137).

\section{Data availability}

All data generated or analyzed during this study are included in this published article and its Supplementary information files.

\section{Conflict of interest}

The authors declare no competing interests.

\section{Ethics statement}

The design and methods of the research are in accordance with the requirements of related and procedures (such as Ethical Review of Biomedical Research involving Human Subject, ICH-GCP) as well as the ethical principles. The study was performed in accordance with the Declaration of Helsinki. The IRB of The Third Xiangya Hospital of Central South University has approved the research to be conducted. The Number of approval for scientific research project is: No. 2019-S493.

\section{Publisher's note}

Springer Nature remains neutral with regard to jurisdictional claims in published maps and institutional affiliations.

Supplementary information The online version contains supplementary material available at https://doi.org/10.1038/s41419-021-03523-z. 
Received: 16 August 2020 Revised: 7 February 2021 Accepted: 9 February 2021

\section{Published online: 04 March 2021}

\section{References}

1. Schadendorf, D. et al. Melanoma. Nat. Rev. Dis. 1, 1-20 (2015).

2. Rastrelli, M., Tropea, S., Rossi, C. \& Alaibac, M. Melanoma: epidemiology, risk factors, pathogenesis, diagnosis and classification. In Vivo 28, 1005-1011 (2014).

3. Lee, C., Thoma, C. \& Ng, K. An overview of the changing landscape of treatment for advanced melanoma. Pharmacotherapy 37, 319-333 (2017).

4. Eggermont, A., Spatz, A. \& Robert, C. Cutaneous melanoma. Lancet 383, 816-827 (2014).

5. Balch, C., Gershenwald, J., Soong, S., Thompson, J. \& Atkins, M. Final version of 2009 AJCC melanoma staging and classification. J. Clin. Oncol. 27, 199-6206 (2009).

6. Mendenhall, W. et al. Adjuvant postoperative radiotherapy for cutaneous melanoma. Acta Oncol. 56, 495-496 (2017).

7. Beadle, B. et al. Radiation therapy field extent for adjuvant treatment of axillary metastases from malignant melanoma. Int. J. Radiat. Oncol. Biol. Phys. 73, 1376-1382 (2009).

8. Mahadevan, A., Patel, V. \& Dagoglu, N. Radiation therapy in the management of malignant melanoma. Oncology 29, 743-751 (2015).

9. Maverakis, E., Cornelius, L., Bowen, G., Phan, T. \& Patel, F. B. Metastatic melanoma-a review of current and future treatment options. Acta Derm. Venereol. 95, 516-524 (2015).

10. Gilkes, D., Semenza, G. \& Wirtz, D. Hypoxia and the extracellular matrix: drivers of tumour metastasis. Nat. Rev. Cancer 14, 430-439 (2014).

11. Barker, H., Paget, J., Khan, A. \& Harrington, K. The tumour microenvironmentafter radiotherapy: mechanisms of resistance and recurrence. Nat. Rev. Cancer 15, 409-425 (2015).

12. Macklin, P., McAuliffe, J., Pugh, C. \& Yamamoto, A. Hypoxia and HIF pathway in cancer and the placenta. Placenta 56, 8-13 (2017).

13. Parks, S., Cormerais, Y. \& Pouysségur, J. Hypoxia and cellular metabolism in tumour pathophysiology. J. Physiol. 595, 2439-2450 (2017).

14. Hanahan, D. \& Weinberg, R. Hallmarks of cancer: the next generation. Cell 144, 646-674 (2011)

15. Nys, K, Maes, H., Dudek, A. \& Agostinis, P. Uncovering the role of hypoxia inducible factor-1alpha in skin carcinogenesis. Biochim. Piophys. Acta 1816 $1-12(2011)$

16. Kuphal, S., Winklmeier, A., Warnecke, C. \& Bosserhoff, A. Constitutive HIF-1 activity in malignant melanoma. Eur. J. Cancer 46, 1159-1169 (2010).

17. Fan, J. et al. Tyrosine phosphorylation of lactate dehydrogenase A is important for NADH/NAD(+) redox homeostasis in cancer cells. Mol. Cell Biol. 31, 4938-4950 (2011).

18. Ke, Q. \& Costa, M. Hypoxia-inducible factor-1 (HIF-1). Mol. Pharmacol. 70 1469-1480 (2006).

19. Meijer, T., Kaanders, J., Span, P. \& Bussink, J. Targeting hypoxia, HIF-1, and tumor glucose metabolism to improve radiotherapy efficacy. Clin. Cancer Res. 18, 5585-5594 (2012).

20. Zhao, F., Ming, J., Zhou, Y. \& Fan, L. Inhibition of Glut1 by WZB117 sensitizes radioresistant breast cancer cells to irradiation. Cancer Chemother. Pharmacol. 77, 963-972 (2016).

21. Shimura, T. et al. AKT-mediated enhanced aerobic glycolysis causes acquired radioresistance by human tumor cells. Radiother. Oncol. 112, 302-307 (2014).

22. Huang, X. et al. HK2 is a radiation resistant and independent negative prognostic factor for patients with locally advanced cervical squamous cell carcinoma. Int. J. Clin. Exp. Pathol. 8, 4054-4063 (2015).

23. Quinn, J. \& Chang, H. Unique features of long non-coding RNA biogenesis and function. Nat. Rev. Genet. 17, 47-62 (2016).

24. Jarroux, J., Morillon, A. \& Pinskaya, M. History, discovery, and classification of IncRNAs. Adv. Exp. Med. Biol. 1008, 1-46 (2017).

25. Xiang, S. et al. LncRNA IDH1-AS1 links the functions of C-Myc and HIF-1a via IDH1 to regulate the Warburg effect. Proc. Natl Acad. Sci. USA 115, 1465-1474 (2018).

26. Yang, F., Zhang, H., Mei, Y. \& Wu, M. Reciprocal regulation of HIF-1a and LINCRNA-p21 modulates the Warburg effect. Mol. Cell. 53, 88-100 (2014).

27. Leonardo, S., Laura, P., Yvonne, T., Lev, K. \& Pier, P. A ceRNA hypothesis: the Rosetta stone of a hidden RNA language? Cell 146, 353-358 (2011).
28. Zhang, P., Cao, L., Fan, P., Mei, Y. \& Wu, M. LncRNA-MIF, a c-Myc-activated long non-coding RNA, suppresses glycolysis by promoting Fbxw7-mediated c-Myc degradation. EMBO Rep. 17, 1204-1220 (2016).

29. Lin, Y. et al. Taurine up-regulated gene 1 functions as a master regulator to coordinate glycolysis and metastasis in hepatocellular carcinoma. Hepatology 67, 188-203 (2018)

30. Leucci, E., Coe, E., Marine, J. \& Vance, K. The emerging role of long non-coding RNAs in cutaneous melanoma. Pigment Cell Melanoma Res. 29, 619-626 (2016).

31. Kabbarah, O. et al. Integrative genome comparison of primary and metastatic melanomas. PLOS ONE 5, 5-2 (2010).

32. Smith, A., Hoek, K. \& Becker, D. Whole-genome expression profiling of the melanoma progression pathway reveals marked molecular differences between nevi/melanoma in situ and advanced-stage melanomas. Cancer Biol. Ther. 4, 1018-1029 (2005).

33. Luan, W. et al. Long noncoding RNA LINC00518 acts as a competing endogenous RNA to promote the metastasis of malignant melanoma via miR-2045p/AP1S2 axis. Cell Death Dis. 10, 855-867 (2019).

34. Oliveira, S., Yang, L., Echevarria-Lima, J., Monteiro, R. Q. \& Rezaie, A. R. Thrombomodulin modulates cell migration in human melanoma cell lines. Melanoma Res. 24, 11-19 (2014).

35. Wang, Z. et al. Association of EGFR gene fragments with nuclear matrix proteins in high and low metastatic melanoma cell lines. Anticancer Res. 19 4349-4352 (1999).

36. Chang, L., Hu, Z., Zhou, Z. \& Zhang, H. Linc00518 contributes to multidrug resistance through regulating the MiR-199a/MRP1 axis in breast cancer. Cell Physiol. Biochem. 48, 16-28 (2018).

37. Wang, D., You, D., Dong, J. \& Liu, F. Knockdown of long non-coding RNA LINC00518 inhibits cenvical cancer proliferation and metastasis by modulating JAKJSTAT3 signaling. Eur. Rev. Med. Pharm. Sci. 23, 496-506 (2019).

38. Zhou, J. et al. MiR-33a functions as a tumor suppressor in melanoma by targeting HIF-1a. Cancer Biol. Ther. 16, 846-855 (2015).

39. Choudhary, C. et al. Lysine acetylation targets protein complexes and coregulates major cellular functions. Science 325, 834-840 (2009).

40. Magnon, C., Opolon, P. \& Schlumberger, M. Radiation and inhibition of angiogenesis by canstatin synergize to induce HIF-1alpha-mediated tumor apoptotic switch. J. Clin. Invest. 117, 1844-1855 (2007).

41. Gerami, P. et al. Development and validation of a noninvasive 2-gene molecular assay for cutaneous melanoma. J. Am. Acad. Dermatol. 76, 114-120 (2017).

42. Ferris, L. et al. Utility of a noninvasive 2-gene molecular assay for cutaneous melanoma and effect on the decision to biopsy. JAMA Dermatol. 153 675-680 (2017).

43. Yu, J. et al. Upregulated long non-coding RNA LINC00152 expression is associated with progression and poor prognosis of tongue squamous cell carcinoma. J. Cancer 8, 523-530 (2017).

44. Zhu, Y. et al. The MRVI1-AS1/ATF3 signaling loop sensitizes nasopharyngeal cancer cells to paclitaxel by regulating the Hippo-TAZ pathway. Oncogene $\mathbf{3 8}$, 6065-6081 (2019).

45. Tay, Y., Rinn, J. \& Pandolfi, P. P. The multilayered complexity of ceRNA crosstalk and competition. Nature 505, 344-352 (2014)

46. Qi, X. et al. CeRNA in cancer: possible functions and clinical implications. J. Med. Genet. 52, 710-718 (510).

47. Chen, H., Du, G., Song, X. \& Li, L. Non-coding transcripts from enhancers: new insights into enhancer activity and gene expression regulation. Genom. Proteom. Bioinform. 15, 201-207 (2017).

48. Moussaieff, A., Rouleau, M., Aberdam, D. \& Nahmias, Y. Glycolysis-mediated changes in acetyl-CoA and histone acetylation control the early differentiation of embryonic stem cells. Cell Metab. 21, 392-402 (2015).

49. Zhang, E., Han, L., Yin, D., Shu, Y. \& Chen, J. H3K27 acetylation activated-long non-coding RNA CCAT1 affects cell proliferation and migration by regulating SPRY4 and HOXB13 expression in esophageal squamous cell carcinoma. Nucleic Acids Res. 45, 3086-3101 (2017).

50. Schoepflin, Z., Shapiro, I. \& Risbud, M. Class I. and Ila HDACs mediate HIFla stability through PHD2-dependent mechanism, while HDAC6, a class Ilb member, promotes HIF-1a transcriptional activity in nucleus pulposus cells of the intervertebral disc. J. Bone Miner. Res. 31, 1287-1299 (2016).

51. Krumm, A. et al. Enhanced histone deacetylase activity in malignant melanoma provokes RAD51 and FANCD2-triggered drug resistance. Cancer Res. 76 3067-3077 (2016). 
52. Han, S. et al. MicroRNA-33a-3p suppresses cell migration and invasion by directly targeting PBX3 in human hepatocellular carcinoma. Oncotarget 7, 42461-42473 (2016)

53. Liang, M., Liu, Z., Lin, H., Shi, B. \& Li, M. Chen T. High-throughput sequencing reveals circular RNA hsa_circ_0000592 as a novel player in the carcinogenesis of gastric carcinoma. Biosci. Rep. 39, 623-641 (2019).

54. Akram, M. et al. Mini-review on glycolysis and cancer. J. Cancer Educ. 28, 454-457 (2013).

55. Ganapathy, S. \& Geschwind, J. Tumor glycolysis as a target for cancer therapy: progress and prospects. Mol. Cancer 12, 52-159 (2013).

56. Görlach, A., Dimova, E., Petry, A. \& Martínez-Ruiz, A. Reactive oxygen species, nutrition, hypoxia and diseases: problems solved? Redox Biol. 6, 372-385 (2015).
57. Nosrati, N., Bakovic, M. \& Paliyath, G. Molecular mechanisms and pathways as targets for cancer prevention and progression with dietary compounds. Int. J. Mol. Sci. 18, 57-63 (2017).

58. Valencak, J., Kittler, H. \& Gonzalez-Inchaurraga, M. Prognostic relevance of hypoxia inducible factor-1alpha expression in patients with melanoma. Clin. Exp. Dermatol. 34, e962-e964 (2009).

59. Zeng, Q. et al. Inhibition of REDD1 sensitizes bladder urothelial carcinoma to paclitaxel by inhibiting autophagy. Clin. Cancer Res. 24, 445-459 (2018).

60. Li, W. et al. TRAF4 is a critical molecule for Akt activation in lung cancer. Cancer Res. 73, 6938-6950 (2013).

61. Franken, N. A., Rodermond, H. M., Stap, J., Haveman, J. \& van Bree, C. Clonogenic assay of cells in vitro. Nat. Protoc. 5, 2315-2319 (2006). 